\title{
Nuclear Magnetic Resonance Spectra of Some Hydrocarbons*
}

\author{
Weston A. ANDERson† \\ CERN, Geneva, Switzerland \\ (Received September 20, 1955)
}

\begin{abstract}
The study of the nuclear magnetic resonance spectrum of protons in ethyl alcohol made by J. T. Arnold has been extended to other liquid compounds which are more amenable to an exact calculation of their energy levels and transition probabilities under the influence of a small radio-frequency perturbation. Resulting recordings of these spectra are shown to be in agreement with the spectra predicted by a simple Hamiltonian proposed by Hahn and Maxwell as well as by Gutowsky, McCall, and Slichter. New evidence has been found which indicates that lines in a given spectrum have different widths.

A method has been demonstrated whereby a definite spin coupling may be associated with a definite group of nuclei. This is achieved by simultaneously applying a large radio-frequency field with a frequency near to the resonance frequency of one group of nuclei and a small radio-frequency field to produce transitions within another group. In this case any structure due to the spin coupling between the two groups, is greatly modified.

A new and simple procedure has been demonstrated whereby the magnitude of the radio-frequency field at the sample may be accurately determined with the aid of a small auxiliary radio-frequency field.
\end{abstract}

\section{INTRODUCTION}

A DETAILED study by Arnold ${ }^{1}$ of the proton magnetic resonance spectrum of ethyl alcohol under a resolution approaching one part in $10^{8}$ has revealed an abundance of fine structure to the resonance lines which had not been previously observed. In this paper the study is extended to other compounds whose spectra are more amenable to an exact analysis than ethyl alcohol. The spectra of these compounds which also show an abundance of fine structure under high resolution are explained by the relatively simple Hamiltonian proposed by Gutowsky, McCall, and Slichter as well as by Hahn and Maxwell. ${ }^{2}$ A perturbation calculation is also presented which when applied to ethyl alcohol is sufficient to explain the many fine details of the spectra observed by Arnold.

Consider a molecular system which contains many identical nuclei. These nuclei may be divided into groups by placing in each group only nuclei with the same chemical shift. The nuclei of a group are said to be "equivalent" if all nuclei in the group have identical coupling to their molecular surroundings. It has been shown $n^{3,4}$ that if the nuclei of a group are "equivalent" then any spin coupling which may exist between them may be omitted from the Hamiltonian of the total system without affecting the calculated transition frequencies or line intensities. In this paper we shall only consider molecules in which all the nuclei within each group are equivalent.

* This work was done in partial fulfillment of the requirements of the degree of Doctor of Philosophy at Stanford University. It was supported in part by the Office of Naval Research and the U. S. Atomic Energy Commission. The material was prepared for publication while working for CERN, Geneva, Switzerland.

$\dagger$ Present address: Varian Associates, Palo Alto, California.

${ }^{1}$ J. T. Arnold, preceding paper [Phys. Rev. 102, 136 (1956)]

2 Gutowsky, McCall, and Slichter, Phys. Rev. 84, 589 (1951);

E. L. Hahn and D. E. Maxwell, Phys. Rev. 84, 1246 (1951).

3 E. L. Hahn and D. E. Maxwell, Phys. Rev. 88, 1070 (1952)

${ }^{4}$ Gutowsky, McCall, and Slichter, J. Chem. Phys. 21, 279 (1953)
In order to calculate the resonance frequencies of a nuclear spin system it is permissible to neglect the effects of weak relaxation ${ }^{5}$ and obtain its stationary energy levels from a suitably chosen Hamiltonian representing the total energy of the system. Throughout this paper we shall measure energy in units of angular frequency by dividing by $\hbar$, the quantum of action divided by $2 \pi$. The proposed Hamiltonian ${ }^{2}$ may be written as

$$
\mathfrak{H C}=-\left\{\sum_{R} \omega_{R} M_{R}+\frac{1}{2} \sum_{R} \sum_{S \neq R} J_{R S} \mathbf{I}_{R} \cdot \mathbf{I}_{S}\right\} .
$$

We have assumed here that we are dealing with different groups of equivalent nuclei. Each group, denoted by a letter $R, S, \cdots$, is characterized by the fact that the shielded magnetic field acting upon the nuclei in the group has the same value so that in the absence of coupling between nuclei it would lead to a common resonance frequency, $\omega_{R}$, for all nuclei in group $R$. The spin operator $\mathbf{I}_{R}$ with the z-component $M_{R}$ chosen in the direction of the applied field has the significance of representing the total spin of the group $R$ and is given by

$$
\mathbf{I}_{R}=\sum_{r} \mathbf{I}_{R r}
$$

Its magnitude can assume all non-negative values $n_{R} I_{R r}, \quad n_{R} I_{R r}-1, n_{R} I_{R r}-2, \cdots$, where $n_{R}$ is the number of nuclei in group $R$ and $I_{R r}$ is the common magnitude of their spin. The constant $J_{R S}$ characterizes the strength of the spin coupling between any nucleus, $r$, in group $R$ and any nucleus, $s$, in group $S$. Since $J_{R S}=J_{S R}$, each term in the sum of Eq. (1) appears twice, which accounts for the factor $1 / 2$ before the sum. The energy levels and transition frequencies of this Hamiltonian are discussed further in Sec. II.

In the study of proton magnetic resonance spectra some evidence has been found indicating that the natural line widths of various lines in the spectra of

\footnotetext{
${ }^{5}$ F. Bloch, this issue [Phys. Rev. 102, 104 (1956)], see Sec. 5.
} 
some chemical compounds are not identical. The question of natural line widths is treated in detail by Bloch in the accompanying paper. However, up to the present time, magnetic field inhomogeneities have prevented accurate measurements of the natural line widths. The evidence of different line widths within a spectrum is presented and discussed in Sec. V.

A method suggested by Bloch ${ }^{6}$ whereby one may identify a spin coupling with a definite group has been demonstrated. The method, which is applicable when the chemical shift is large compared to the corresponding spin coupling, consists in affecting one group of nuclei with a relatively high radio-frequency power while simultaneously observing the other group with a small radio-frequency field. The spacing of the different resonance lines, produced by the spin coupling between the two groups is then greatly reduced.

One may see in a qualitative way how this reduction of the spacing arises from the following argument: Consider a molecule with two groups of nuclei denoted by $A$ and $B$. Assume that one applies a large radiofrequency magnetic field of magnitude $H_{1}$, rotating in the $x-y$ plane with angular frequency $\omega_{1} \cong \omega_{A}$; simultaneously the transition frequencies in the vicinity of $\omega_{B}$ are investigated by producing resonance with a weak radio-frequency field of magnitude $\mathrm{H}_{2}$ and of angular frequency $\omega_{2} ; H_{2}$ shall be small enough so that it does not appreciably perturb the stationary states of group $B$. The problem may be reduced to a time independent one by transforming to a coordinate system rotating with angular frequency $\omega_{1}$. It can be shown ${ }^{7}$ that the effective field in a system rotating with angular velocity $\omega_{1}$ is equal to the field in the laboratory system reduced by the resonance field $\omega_{1} / \gamma$. Including the contribution by the radio-frequency field $H_{1}$ in the rotating frame where it is assumed to point in the $x$-direction, the Hamiltonian is then given by

$$
\begin{aligned}
\mathcal{H C}=-\left\{\left(\omega_{A}-\omega_{1}\right) M_{A}\right. & +\gamma H_{1} I_{A x} \\
& \left.+\left(\omega_{B}-\omega_{1}\right) M_{B}+J_{A B} \mathbf{I}_{A} \cdot \mathbf{I}_{B}\right\} .
\end{aligned}
$$

It may be seen that if $\omega_{1} \cong \omega_{A}$ and $\left|\gamma H_{1}\right| \gg\left|J_{A B}\right|$, the effective field seen by the nuclei in group $A$ is predominantly in the $x$-direction and thus the average value of the total spin $\mathbf{I}_{A}$ will lie essentially in this direction. Also if $\left|\omega_{B}-\omega_{A}\right| \gg\left|J_{A B}\right|$ then the effective field seen by nuclei in group $B$ will be predominantly in the $z$-direction, so that the average value of the scalar product is nearly zero. The last term in Eq. (3) can, therefore, be neglected and the corresponding splitting in this approximation disappears.

Bloom and Shoolery ${ }^{8}$ have presented an approximate treatment, valid in the limit of large separation of the resonance frequency between two groups; while con-

\footnotetext{
${ }^{6}$ F. Bloch, Phys. Rev. 93, 944(A) (1954).

${ }^{7}$ Rabi, Ramsey, and Schwinger, Revs. Modern Phys. 26, 167 (1954).
}

${ }^{8}$ A. L. Bloom and J. N. Shoolery, Phys. Rev. 97, 1261 (1955). firming the above conclusion in the limit of large radiofrequency amplitudes, they show that for intermediate radio-frequency amplitudes the multiplet structure of the spectrum of the undisturbed nuclear group often increases in complexity. A consistant theory of the effect has been presented by Bloch ${ }^{9}$ which takes into account the modification by the strong radio-frequency field of the line width, intensity, and frequency of the resonances in the weak radio-frequency field. He shows that in the limit of large radio-frequency amplitudes one may still have a residual splitting of the order of $J^{2} / \delta$ where $J$ is the spin coupling constant and $\delta$ the corresponding chemical shift.

The apparatus and the experimental techniques employed in these double irradiation experiments are more fully described in Sec. III and Sec. IV, respectively. The technique is demonstrated with several chemical compounds in Sec. V.

As pointed out in Sec. VII of the accompanying paper by Bloch the principle of simultaneous application of a strong and a weak radio-frequency field with different frequencies $\omega_{1}$ and $\omega_{2}$, respectively, can also be applied to a spin system where all nuclei are equivalent and can here be used for calibrating the amplitude of the strong radio-frequency field purely in terms of measured frequencies. This principle of calibration has been successfully applied here.

The fact that there are two values of the resonance frequency $\omega_{2}$ of the weak field, satisfying the equation

$$
\left(\omega_{2}-\omega_{1}\right)^{2}=\left(\omega_{A}-\omega_{1}\right)^{2}+\left(\gamma H_{1}\right)^{2},
$$

is shown in Appendix I by the phenomenological equations. Qualitatively, it can be derived by considering the spin system in a coordinate frame rotating with the angular velocity $\omega_{1}$ of the strong radio-frequency field. In this case the Hamiltonian in the rotating frame is given by Eq. (3) upon omitting the terms associated with group $B$.

$$
\mathfrak{H}=-\left\{\left(\omega_{A}-\omega_{1}\right) M_{A}+\gamma H_{1} I_{A x}\right\} .
$$

The magnitude of the effective magnetic field in the rotating frame is

$$
H_{\text {eff }}=\left\{\left(\gamma H_{1}\right)^{2}+\left(\omega_{A}-\omega_{1}\right)^{2}\right\}^{\frac{1}{2}} / \gamma,
$$

and the magnetic moment can therefore perform a free precession with angular frequency $\gamma H_{\text {eff }}$. As shown in Fig. 1, the projection of this precession upon the $x^{\prime}-y^{\prime}$ plane of the rotating frame describes an ellipse and can be decomposed into two components of different magnitude rotating in opposite directions with the same angular frequency $\gamma H_{\text {eff. }}$. Going back into the laboratory system the free precession contains therefore two frequencies, $\omega_{1} \pm \gamma H_{\text {eff. }}$. The angular frequency of the weak radio-frequency field, $\omega_{2}$, must be made equal to one of these frequencies in order to produce resonance, and consequently one obtains the resonance condition given by Eq. (4).

${ }^{9}$ F. Bloch, reference 5, Sec. 7, in particular see Eq. (7.121). 
Experimentally it is convenient to hold $\omega_{1}$ and $\omega_{2}$ fixed while $\omega_{A}$ is continuously varied. It must be noted that Eq. (4) is likewise satisfied for two values of $\omega_{A}$ if $\left(\omega_{2}-\omega_{1}\right)^{2}>\left(\gamma H_{1}\right)^{2}$. By observing the successive occurrence of both resonances and extrapolating to their coincidence for $\left(\omega_{2}-\omega_{1}\right)^{2}=\left(\gamma H_{1}\right)^{2}$, it is thus possible to determine $H_{1}$. The experimental procedure is discussed in Sec. IV.

In practice the small auxiliary radio-frequency field may also be realized by either an amplitude modulation of the applied magnetic field or by a frequency or amplitude modulation of the radio-frequency field. For a small index of modulation any of these methods produce essentially two sidebands placed symmetrically about the strong radio-frequency field. The resonance condition of the sidebands is thus given by

$$
\omega^{2}=\left(\omega_{A}-\omega_{1}\right)^{2}+\left(\gamma H_{1}\right)^{2},
$$

where $\omega^{\prime}$ is the frequency of the modulation.

\section{CALCULATIONS OF TRANSITION FREQUENCIES AND LINE INTENSITIES}

We shall in this paper consider the cases where each group contains only equivalent nuclei. The Hamiltonian is then given by Eq. (1). By defining the operators $M_{R^{ \pm}}^{ \pm}=I_{R x} \pm i I_{R y}$ the Hamiltonian may be written in the following form:

$$
\mathcal{H C}=-\sum_{R}\left\{\omega_{R} M_{R}+\frac{1}{2} \sum_{S \neq R} J_{R S}\left(M_{R} M_{S}+M_{R}+M_{S^{-}}\right)\right\} .
$$

Using the well-known matrix elements ${ }^{10}$ for $M_{R}$ and $M_{R}^{ \pm}$in the representation in which $\left(\mathbf{I}_{R}\right)^{2}$ and $M_{R}$ are diagonal with eigenvalues $I_{R}\left(I_{R}-1\right)$ and $m_{R}$, respectively, the matrix elements of the Hamiltonian (8) are given by

$$
\begin{aligned}
\left(m_{A}, m_{B}, \cdots|\mathfrak{H C}| m_{A}, m_{B}, \cdots\right) \\
\quad=-\sum_{R=A, B, \cdots}\left\{\omega_{R} m_{R}+\frac{1}{2} \sum_{S \neq R} J_{R S} m_{R} m_{S}\right\},
\end{aligned}
$$

and

$$
\begin{array}{r}
\left(\cdots m_{S}, m_{T}, \cdots|\mathfrak{H}| \cdots m_{S}-1, m_{T}+1, \cdots\right) \\
=-\frac{1}{2} J_{S T}\left\{\left(I_{S}-m_{S}+1\right)\left(I_{S}+m_{S}\right)\left(I_{T}-m_{T}\right)\right. \\
\left.\times\left(I_{T}+m_{T}+1\right)\right\}^{\frac{1}{2}},
\end{array}
$$

with all other matrix elements of $\mathcal{H C}$ equal to zero. Since according to Eq. (10) there exist nonvanishing matrix elements only between states for which one of the quantum numbers $m_{S}$ differs by \pm 1 while another quantum number $m_{T}$ differs by $\mp 1$, it is seen that $m=\sum_{R} m_{R}$ is a good quantum number. The eigenvalues of the matrix given by (9) and (10) shall be denoted by $E(k)$ and the corresponding diagonalizing spin functions by $v(k)$.

${ }^{10}$ See for example L. I. Schiff, Quantum Mechanics (McGrawHill Book Company, Inc., New York, 1949), first edition, p. 143.
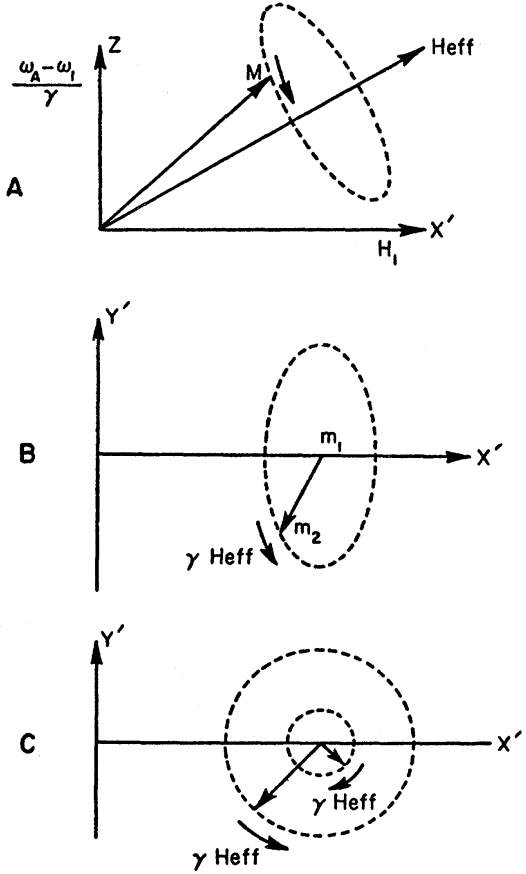

FIG. 1. (A) In the coordinate frame rotating with the frequency of the large radio-frequency field, the magnetic moment carries out a free precession about the effective field $H_{\text {eff }}$ with an angular frequency $\gamma H_{\text {eff. }}$ (B) The projection of this magnetic moment on the $x^{\prime}-y^{\prime}$ axis of the rotating frame describes an ellipse. (C) This ellipse is decomposed into two components of different magnitudes rotating with the same angular frequency but in opposite directions.

The perturbing Hamiltonian $\mathfrak{H}^{\prime}$ due to the radiofrequency field in the $x$-direction may be expressed as

$$
\mathcal{F C}^{\prime}=-\gamma H_{1} \cos \left(\omega_{1} t\right) \sum_{R}\left(M_{R^{+}}+M_{R^{-}}\right) .
$$

Resonance occurs when the frequency $\omega_{1}$, of the radiofre quency field satisfies the condition $\pm \omega_{1}=E(i)-E(j)$. Under the conditions met in this investigation one can use the result derived by Bloch ${ }^{11}$ that the integrated intensity of each resonance line is proportional to the absolute square of the corresponding matrix element of $M^{+}=\sum_{R} M_{R}^{+}$or $M^{-}=\sum_{R} M_{R}^{-}$. The calculation of the maximum height of the lines poses a far more difficult problem in so far as it involves a knowledge of the line widths. However, to the extent to which one may assume the widths of the different resonance lines in a spectrum to be equal, one is allowed to use the expression for their integrated intensity also as a measure for the maximum line height.

The calculation of the transition frequencies and line intensities are particularly simple if only two chemical groups are present and if one of the groups has a maximum spin of $\frac{1}{2}$. In this case one need only solve quadratic equations to find the energy levels. ${ }^{12}$ The simplest case is that of two groups of nuclei with each

\footnotetext{
$11 \mathrm{~F}$. Bloch, reference 5, see Eq. (5.10).

12 Banerjee, Das, and Saha, Proc. Roy. Soc. (London) A226, 490 (1954).
} 
TABLE I. Theoretical transition frequencies and relative line intensities for two groups of nuclei with spin $\frac{1}{2}$ with one nucleus in each group. The chemical shift is denoted by $\delta=\omega_{A}-\omega_{B}$ and the spin coupling constant by $J$.

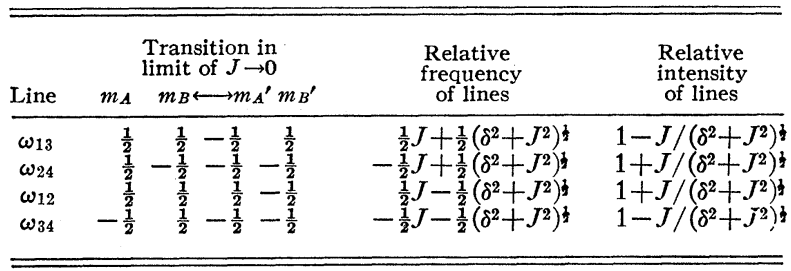

group containing only one nucleus of spin $\frac{1}{2}$. The transition frequencies and line intensities for this case were first given by Hahn and Maxwell ${ }^{3}$ and are summarized in Table I. In Sec. V, experimental spectra of two different chemical compounds are compared with these results. The transition frequencies and intensities for the case of two chemical groups with one proton in one group and two protons in the other group has also been treated by Hahn and Maxwell. Results of this calculation are summarized in Table II and compared with experiment in Sec. V.

By using the procedure outlined above, numerical calculations have been made for two special cases, that of $\beta$-propiolactone which has two protons in each of two groups and that of ethyl bromide which has two protons in one group and three protons in the other. The results for these molecules are given in Tables III and $\mathrm{V}$ respectively, which are found in Sec. V.

A perturbation calculation is indicated when the spin coupling parameter is small compared to the chemical shift. Such a calculation keeping terms up to third order in the energy levels and to first order in the transition probabilities is given in Appendix II. The calculation is made for an arbitrary number of chemical groups and each group with an arbitrary total spin. However for the purposes of discussion it is convenient to consi.les chemical compounds which have only two groups, $A$ and $B$, of equivalent nuclei. The Hamiltonian in this case is

$$
\mathscr{H}=-\left\{\omega_{A} M_{A}+\omega_{B} M_{B}+J_{A B} \mathbf{I}_{A} \cdot \mathbf{I}_{B}\right\} .
$$

Gutowsky, McCall, and Slichter ${ }^{4}$ have shown that for $\left|J_{A B}\right| \ll\left|\omega_{A}-\omega_{B}\right|$ one obtains in first approximation for the energy levels of the spin system

$$
E=-\left\{\omega_{A} m_{A}+\omega_{B} m_{B}+J_{A B} m_{A} m_{B}\right\}
$$

where $m_{A}, m_{B}$ are the eigenvalues of $M_{A}, M_{B}$. The selection rules of transitions are $\Delta I_{A}=\Delta I_{B}=0$ and further in this approximation $\Delta m_{A}= \pm 1, \Delta m_{B}=0$, or $\Delta m_{A}=0, \Delta m_{B}= \pm 1$.

Considering transitions of the nuclei in group $A$, we note that for a given total spin $I_{A}$ there are $\left(2 I_{A}+1\right)$ equally spaced energy levels with spacing equal to the transition frequency $\omega_{A}+J_{A B} m_{B}$. Since $m_{B}$ can assume $2\left(I_{B}\right)_{\max }+1$ different values, there are this many different spectrum lines associated with transitions in group $A$ with their frequencies separated by the amount
$J_{A B}$. The intensity of each line is proportional to the statistical weight of the corresponding value of $m_{B}$. Levels which differ only in the total spin quantum numbers $I_{A}, I_{B}$, are degenerate in this approximation.

In Appendix II the energy terms in second order are calculated with the result that for the case of two groups of equivalent nuclei one has to add to the expression given in Eq. (12) the term ${ }^{13}$

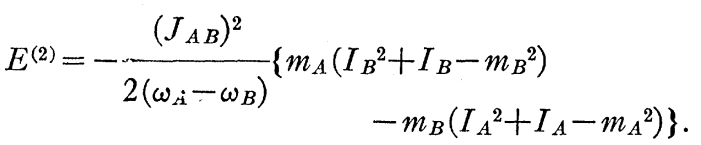

In this approximation the $2 I_{A}+1$ energy levels of group $A$ are no longer equally spaced since there is a term proportional to $m_{A}{ }^{2}$. Thus for a given assignment of the total spin quantum numbers $I_{A}$ and $I_{B}$ each line of group $A$ is further split into $2 I_{A}$ lines, the separation being of the order of $\left(J_{A B}\right)^{2} /\left(\omega_{A}-\omega_{B}\right)$. For the case of $m_{B}=0$ there is an exception to this rule and the levels

TABLE II. Theoretical transition frequencies and relative line intensities for two groups of nuclei of spin $\frac{1}{2}$ with one nucleus in group $A$ and two nuclei in group $B$. The chemical shift is denoted by $\delta=\omega_{A}-\omega_{B}$, and the spin coupling constant by $J$. $\omega_{A}$ is arbitrarily called zero on the frequency scale and the

\begin{tabular}{|c|c|c|c|c|c|}
\hline \multirow{3}{*}{$\begin{array}{r}m_{A} \\
\frac{1}{2}\end{array}$} & \multicolumn{3}{|c|}{$\underset{J \rightarrow 0}{\text { Transition in limit }}$} & $\begin{array}{c}\text { Transition } \\
\text { frequency } \\
\text { measured } \\
\text { from } \omega_{A}\end{array}$ & $\begin{array}{l}\text { Relative line } \\
\text { intensity }\end{array}$ \\
\hline & \multirow{2}{*}{1} & \multirow{2}{*}{$-\frac{1}{2}$} & \multirow{2}{*}{1} & \multirow{2}{*}{$-\frac{1}{2} \delta+\frac{1}{2} J+S$} & $(1-2 T)^{2}$ \\
\hline & & & & & $1+2 T^{2}$ \\
\hline \multirow{2}{*}{$\frac{1}{2}$} & \multirow{2}{*}{0} & \multirow{2}{*}{$-\frac{1}{2}$} & \multirow{2}{*}{0} & \multirow{2}{*}{$-\delta+S+S^{\prime}$} & $\left(1-2 T^{\prime}+2 T\right)^{2}$ \\
\hline & & & & & $\left(1+2 T^{2}\right)\left(1+2 T^{\prime 2}\right)$ \\
\hline$\frac{1}{2}$ & $0^{\text {a }}$ & $-\frac{1}{2}$ & 0 & 0 & 1 \\
\hline \multirow{2}{*}{$\frac{1}{2}$} & \multirow{2}{*}{-1} & \multirow{2}{*}{$-\frac{1}{2}$} & \multirow{2}{*}{-1} & \multirow{2}{*}{$\frac{1}{2} \delta-\frac{1}{2} J+S^{\prime}$} & $\left(1+2 T^{\prime}\right)^{2}$ \\
\hline & & & & & $1+2 T^{\prime 2}$ \\
\hline \multirow{2}{*}{$\frac{1}{2}$} & \multirow{2}{*}{0} & \multirow{2}{*}{$\frac{1}{2}$} & \multirow{2}{*}{-1} & \multirow{2}{*}{$-\delta-J+S-S^{\prime}$} & $2\left(1+T^{\prime}+2 T T^{\prime}\right)^{2}$ \\
\hline & & & & & $\left(1+2 T^{2}\right)\left(1+2 T^{\prime 2}\right)$ \\
\hline \multirow{2}{*}{$\frac{1}{2}$} & \multirow{2}{*}{1} & \multirow{2}{*}{$\frac{1}{2}$} & \multirow{2}{*}{0} & \multirow{2}{*}{$-\frac{1}{2} \delta+J-S$} & $2(1+T)^{2}$ \\
\hline & & & & & $1+2 T^{2}$ \\
\hline \multirow{2}{*}{$-\frac{1}{2}$} & \multirow{2}{*}{1} & \multirow{2}{*}{$-\frac{1}{2}$} & \multirow{2}{*}{0} & \multirow{2}{*}{$-\delta+J-S+S^{\prime}$} & $2\left(1-T+2 T T^{\prime}\right)^{2}$ \\
\hline & & & & & $\left(1+2 T^{2}\right)\left(1+2 T^{\prime 2}\right)$ \\
\hline \multirow{2}{*}{$-\frac{1}{2}$} & \multirow{2}{*}{0} & \multirow{2}{*}{$-\frac{1}{2}$} & & & $2\left(1-T^{\prime}\right)^{2}$ \\
\hline & & & -1 & $-\frac{1}{2} \delta-J-S$ & $1+2 T^{\prime 2}$ \\
\hline & & & & & $4\left(T^{\prime}-T-T T^{\prime}\right)^{2}$ \\
\hline-2 & 1 & -2 & -1 & $-0-2-n$ & $\left(1+2 T^{2}\right)\left(1+2 T^{\prime 2}\right)$ \\
\hline
\end{tabular}
abbreviations $S=\frac{1}{4} J+\frac{1}{2}\left(\delta^{2}+\delta J+9 J^{2} / 4\right)^{\frac{1}{2}}, \quad S^{\prime}=-\frac{1}{4} J+\frac{1}{2}\left(\delta^{2}-\delta J\right.$ $\left.+9 J^{2} / 4\right)^{\prime}, T=J /(\delta+2 S)$, and $T^{\prime}=J /\left(\delta+2 S^{\prime}\right)$ are used.

a Transition with $I_{B}=0$, all others with $I_{B}=1$.

${ }^{13}$ This result has also been derived by $F$. Bloch, this issue [Phys. Rev. 102, 104 (1956)], see Eq. (6.36). 
FIG. 2. Block diagram of the double irradiation transmitter system.

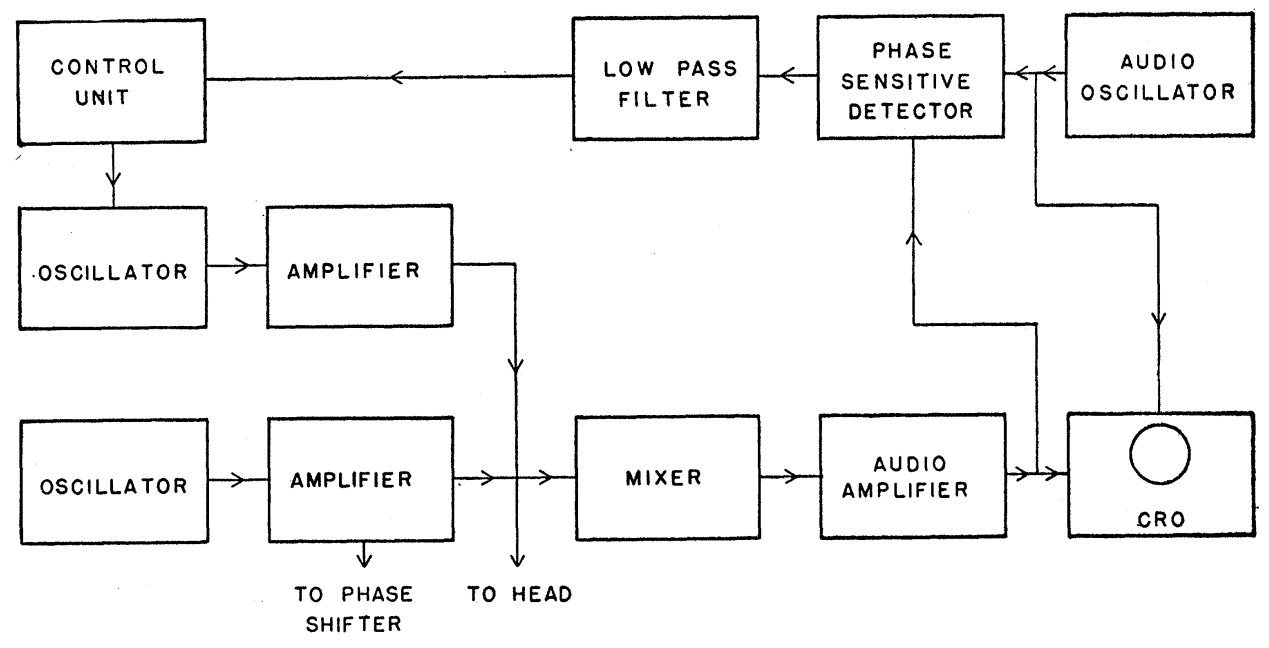

of group $A$ are still equally spaced. However if the total spin quantum number $I_{B}$ may assume more than one value, the transitions with different total spins $I_{B}$ will have different frequencies; these separations are also of the order of $\left(J_{A B}\right)^{2} /\left(\omega_{A}-\omega_{B}\right)$, while two transitions described by a set of quantum numbers which differ only in $I_{A}$, still have the same frequency.

Including second-order terms the frequency of a transition between a state with energy $E\left(m_{A}, m_{B}\right)$ and another with energy $E\left(m_{A}-1, m_{B}\right)$ is

$$
\begin{aligned}
\omega=\omega_{A}+J_{A B} m_{B} & +\frac{J_{A B}{ }^{2}}{2\left(\omega_{A}-\omega_{B}\right)} \\
& \times\left\{I_{B}\left(I_{B}+1\right)-m_{B}\left(m_{B}+1\right)+2 m_{A} m_{B}\right\} .
\end{aligned}
$$

It is of interest to note that the spectrum of a chemical compound does not allow the determination of the sign of the spin coupling constant. However if a chemical group is coupled to two other groups and if the spectrum is sufficiently well resolved to be able to observe the effects predicted by second-order perturbation theory, then the relative sign of the two spin coupling constants may be determined.

The energy corrections to third order have been calculated with the results given in Appendix II. In third order almost all transition lines are described by a unique set of quantum numbers. One exception arises in the special case where the total spin $I_{B}=0$. Of course in this case the levels of group $A$ are equally spaced even in an exact analysis.

The transition probabilities between two states of the nuclear spin system under resonance conditions when a small radio-frequency perturbation is applied, are calculated (see Appendix II) using for the initial and final wave functions the expressions obtained in the first approximation. For the case of two groups of equivalent nuclei, the signal intensity for a transition in group $A$ between the energy levels associated with the quantum numbers $m_{A}$ and $m_{A}-1$ is found to be proportional to

$$
\left(I_{A}-m_{A}+1\right)\left(I_{A}+m_{A}\right)\left\{1-\frac{2 J_{A B}}{\omega_{A}-\omega_{B}} m_{B}\right\} .
$$

We note that in a given multiplet the lines with a frequency further away from those of the group which causes the splitting have been reduced in intensity while the lines closer to this group have greater intensity. This fact may be of some help in identifying spectra of complicated molecules, provided that the chemical shift is not too large compared to the corresponding spin coupling.

\section{APPARATUS}

The apparatus used in obtaining high-resolution recordings is described by Arnold. ${ }^{1}$ When double irradiation experiments were performed the oscillator and amplifier shown in Fig. 5 of Arnold's paper were replaced by the double transmitter system shown in Fig. 2. The two oscillators were crystal controlled and operated at different submultiples of the final frequency in order to avoid "pulling" due to coupling between them. The output of the oscillators was multiplied, one by four, the other by five, in the amplifiers. The power output of the two amplifiers could be varied independently and was fed into the nuclear resonance head.

It is well known that a crystal oscillator may be "pulled" up to about $0.01 \%$ by placing a suitable reactance in the crystal circuit. One of the two crystal oscillators was, therefore, controlled by a variable reactance circuit in such a manner that the frequency difference of the two oscillators after multiplication was maintained at a fixed value. This was accomplished by mixing the outputs of the two amplifiers to obtain their difference frequency. This difference frequency was then fed into a phase-sensitive detector whose reference was derived from an audio-oscillator. When the difference frequency was equal to the frequency of the audio-oscillator, the output voltage of the phase 
detector was determined by the phase difference of the two frequencies. This output voltage was filtered to remove the audio-frequency components. It was then fed into the reactance circuit which controlled the radio-frequency oscillator in such a manner as to tend to keep the phase difference at a constant value.

It was possible to ascertain whether the frequency difference was actually maintained by observing a cathode-ray oscilloscope which had the mixer voltage applied to the vertical axis and the audio-oscillator voltage applied to the horizontal axis. A nonrotating ellipse was observed under proper operating conditions. The radio-frequency oscillator could be brought within the controlling range of the control circuit by means of a variable capacitor connected in the crystal circuit of one of the oscillators.

In the double-irradiation experiments the variable reactance circuit was used to control the frequency of the stronger of the two radio-frequency outputs. The reference voltage for the receiver phase detector was derived from the weaker of the two radio-frequency outputs.

\section{EXPERIMENTAL PROCEDURE}

\section{A. High-Resolution Traces}

The high-resolution traces were taken under slow passage conditions and with sufficiently low radiofrequency power to avoid saturation. They were recorded on paper tape with a Sanborn Model 127 recorder. A standard tape speed of 1 millimeter per second was used, although a few traces of the complete spectrum of a compound were made at a speed of $\frac{1}{2}$ millimeter per second. The magnetic field was always swept from high to low field so that resonances which occur at the higher magnetic field appear to the left side of the trace. The radio-frequency was fixed at about $30.5 \mathrm{Mc} / \mathrm{sec}$. The traces would appear the same if the magnetic field were fixed and the radio-frequency field swept toward higher frequencies toward the right side of the trace.

Only the spectra of protons in liquid samples have been studied. The observed width of most of the resonance lines of the various compounds had to be attributed to inhomogeneities of the applied magnetic field.

\section{B. Calibration of Traces}

One of the simplest methods of calibrating the frequency difference of the various resonances of a spectrum consisted in applying a sinusoidal modulation of the applied field. It is shown in Appendix III that if if the amplitude of the radio-frequency field is far below saturation, a series of equally spaced resonance lines appear for each resonance line in the unmodulated spectrum. The frequency spacing between corresponding lines is equal to the frequency of the modulating field and thus these lines serve as a calibration for the spectra lines.
The amplitude of the sideband resonances depends upon the magnitude of the modulation and upon the mode of detection. The resonance signal of each sideband contains the transmitter frequency and all frequencies which differ from it by an integral multiple of the modulation frequency. Since the phase-sensitive detector in the receiver derived its reference voltage from the transmitter, only the component which had the same frequency as the transmitter appeared as a direct current signal in the output, the other components were removed by filtering. It is shown in Appendix III that under these conditions the $n$th sideband has a relative amplitude of $J_{n}^{2}\left(\omega_{m} / \omega^{\prime}\right)$, where $\omega_{m}$ is the peak amplitude of the modulating field measured in units of angular frequency by multiplying the field by $\gamma ; \omega^{\prime}$ is the angular frequency of the modulating field, and $J_{n}\left(\omega_{m} / \omega\right)$ is a Bessel function of the first kind.

\section{Double Irradiation Experiments}

In some cases it is desirable to affect strongly one group of nuclei while observing another group with a small radio-frequency field. In the approximation of the chemical shift large compared to the spin coupling any structure due to the spin coupling with the strongly affected group disappears, leaving only structures which may exist due to spin couplings with other groups.

The experiment was performed by keeping fixed the difference between the frequencies of the strong and of the weak radio-frequency fields and sweeping the magnetic field. The reference voltage for the phasesensitive detectors was derived from the source of the weak radio-frequency field so that only transitions due to the weak radio-frequency field were observed as a direct current signal on the trace. The signal from the strong radio-frequency field appeared as an alternating voltage of a frequency equal to the difference frequency of the two transmitters.

\section{Calibration of the Radio-Frequency Field}

An indication of the magnitude of the strong radiofrequency field could be obtained by measuring the direct current voltage which was produced by a crystal diode connected to the input of the head. However, since the diode output voltage was an unknown function of the radio-frequency voltage, it could not be used for calibration, which was carried out by the following procedure.

A water sample with the proton resonance lines broadened by the addition of paramagnetic salts was placed in the head and the signal height was plotted as a function of the diode output voltage. The measurement was carried out far below saturation so that the signal height could be expected to be proportional to the magnitude of the radio-frequency field.

The absolute magnitude of the radio-frequency field was determined by a double irradiation experiment (see 
Sec. I) using a distilled-water sample. Let $\omega_{1}$ and $H_{1}$ be the angular frequency and magnitude of the strong field to be measured. The weak field shall have an angular frequency $\omega_{2}$ and it shall be of sufficiently low amplitude so as not to perturb the system appreciably. Actually, it was the difference $\omega_{2}-\omega_{1}$ which was kept closely constant and determined by an audio-oscillator. As pointed out in Sec. I and shown in Appendix I the resonance condition for the weak radio-frequency field is given by $\left(\omega_{2}-\omega_{1}\right)^{2}=\left(\omega_{A}-\omega_{1}\right)^{2}+\left(\gamma H_{1}\right)^{2}$. In the measurements the magnetic field was swept linearly with time which corresponds to a linear change of the resonance frequency $\omega_{A}$. For $\left(\omega_{2}-\omega_{1}\right)^{2}>\left(\gamma H_{1}\right)^{2}$, the resonance equation above is satisfied for two different values of $\omega_{A}$ which are situated symmetrically about
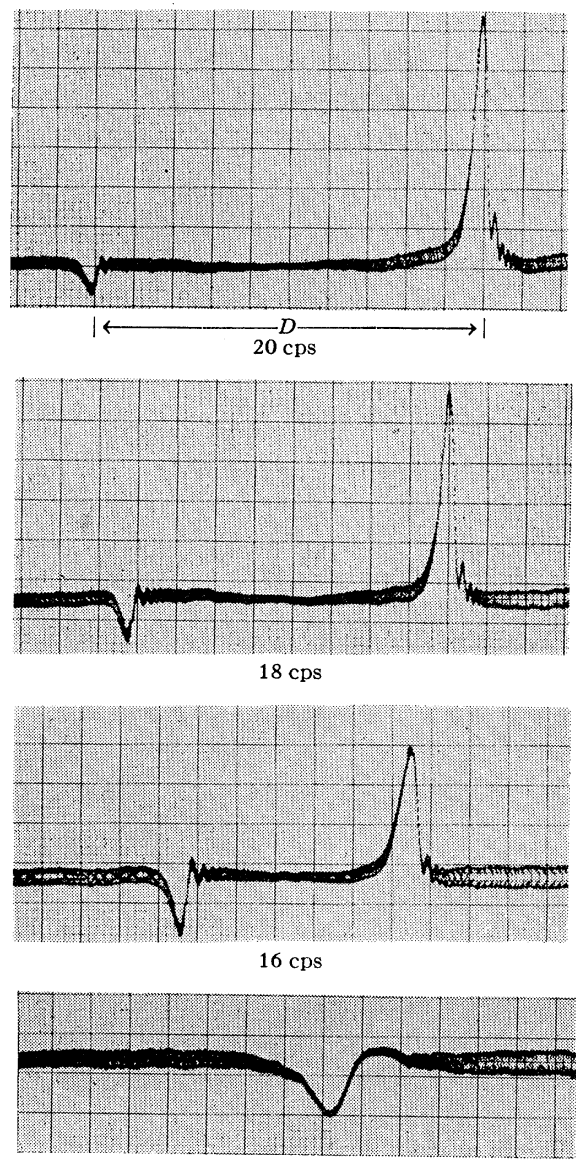

$14 \mathrm{cps}$

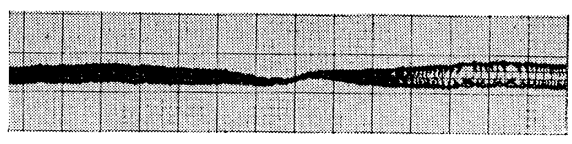

$13.5 \mathrm{cps}$

FIG. 3. Transitions produced by a small radio-frequency field of angular frequency $\omega_{2}$ when water was simultaneously irradiated by a large radio-frequency field of angular frequency $\omega_{1}$. The number below each trace is the frequency difference of the two fields $\left(\omega_{2}-\omega_{1}\right) /(2 \pi)$. The relative magnitude of the two signals is here immaterial and distorted since the receiver was overoaded by the large radio-frequency field.

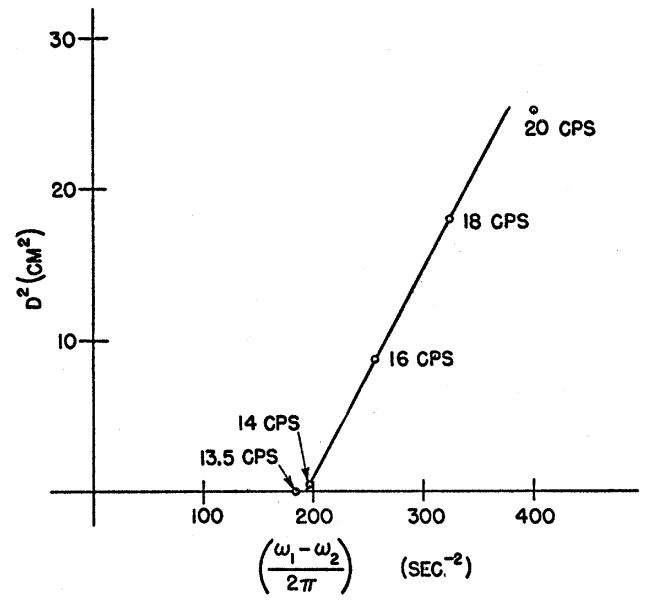

Fig. 4. Plot of the square of the distance $D$, between resonances of each trace shown in Fig. 3, using the square of the difference frequency $\left(\omega_{2}-\omega_{1}\right) /(2 \pi)$ as the abscissa. Each point is labeled by the frequency difference of the two fields, $\left(\omega_{2}-\omega_{1}\right) /(2 \pi)$.

the frequency $\omega_{1}$ of the strong field. If the magnitude of the difference $\omega_{2}-\omega_{1}$ is decreased in successive traces, the two resonances appear closer together. At the limiting value of $\left(\omega_{2}-\omega_{1}\right)^{2}=\left(\gamma H_{1}\right)^{2}$, the two resonances coalesce, and for differences $\omega_{2}-\omega_{1}$ smaller than this amount no resonance is observed. Such a series of traces is shown in Fig. 3. Below each trace is given the difference frequency $\left(\omega_{2}-\omega_{1}\right) / 2 \pi$ of the strong and weak fields. The square of the distance $D$ on the trace between resonances for different values of $\left(\omega_{2}-\omega_{1}\right) / 2 \pi$ has been plotted in Fig. 4 using $\left(\omega_{2}-\omega_{1}\right)^{2} /(2 \pi)^{2}$ as the abscissa, the data being taken from the traces shown in Fig. 3. To the extent to which the sweep field varies linearly with time and has the same rate of change for all the traces the points on this graph should lie along a straight line, intersecting the abscissa axis at $\left(\gamma H_{1}\right)^{2} /(2 \pi)^{2}$. This fact has been ascertained within the experimental error, yielding in this case the value $H_{1}=3.5 \pm 0.2$ milligauss.

It is interesting to note that when the difference frequency $\omega_{2}-\omega_{1}$ is made larger, one of the resonance lines becomes weaker and the other becomes stronger. In the limit of a very large difference frequency, one of the resonances disappears and the other resonance fulfills the usual resonance condition $\omega_{2}=\omega_{A}$.

The absolute magnitude of the radio-frequency field has also been determined by applying magnetic field modulation in place of the weak radio-frequency field. In this case the index of modulation was made sufficiently small so that the resonances of the sidebands do not perturb the system appreciably. Under these conditions only the first sidebands produce observable signals. The frequency of these signals differs from the transmitter frequency by the modulating frequency, $\omega^{\prime}$, and thus these signals appear as an alternating voltage at the output of the phase-sensitive detector. The resonance condition for the sidebands is given by 
<smiles>ClC12C=CC1(Br)S2</smiles>

\section{2- BROMO-5-CHLOROTHIOPHENE}
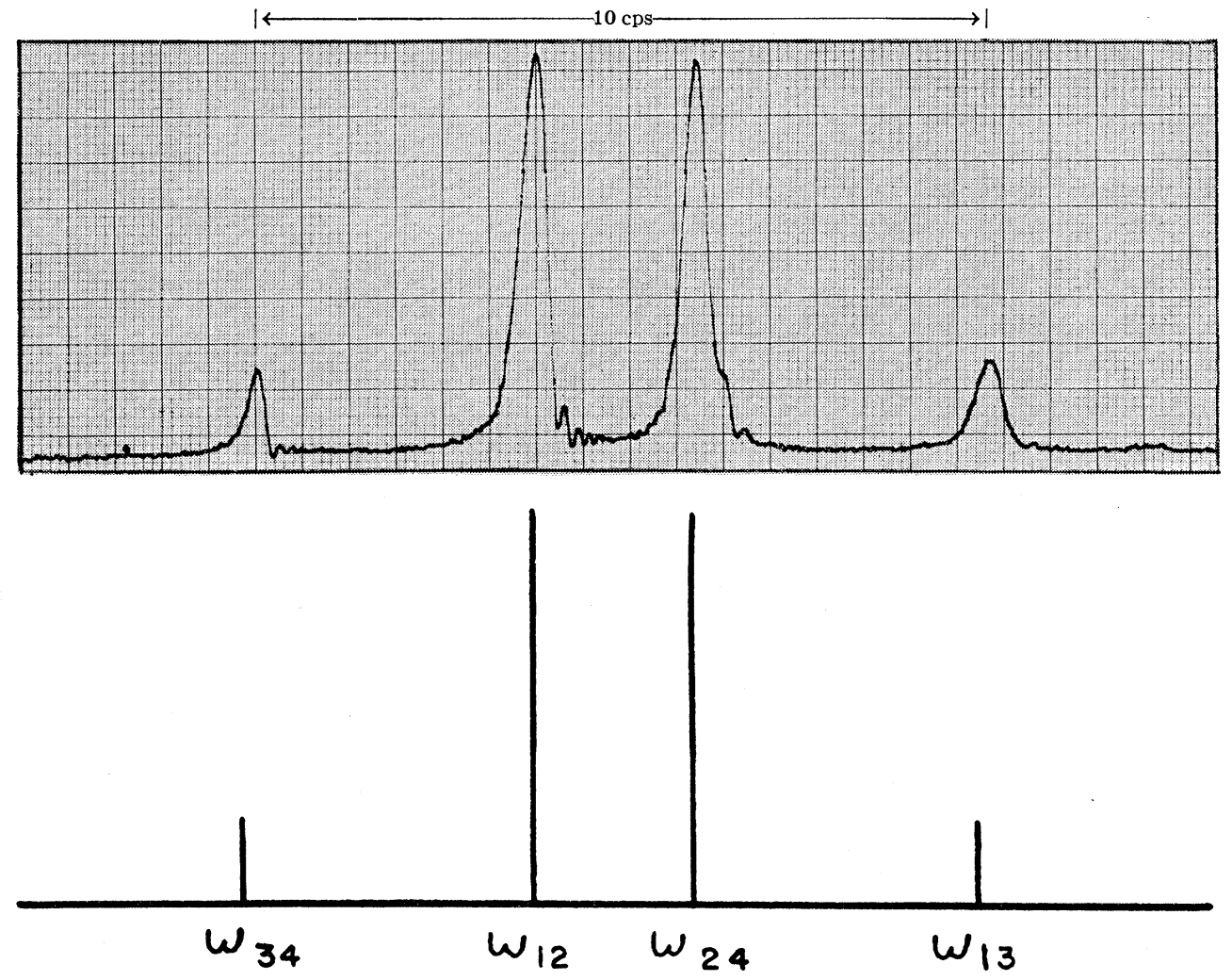

Fig. 5. Spectrum of 2-bromo-5-chlorothiophene. The theoretical spectrum below was calculated from the expressions given in Table I with the ratio $J / \delta=0.835$. The labeling of the lines is identical to that given in Table I.

$\omega^{\prime 2}=\left(\omega_{A}-\omega_{1}\right)^{2}+\left(\gamma H_{1}\right)^{2}$. Thus if one plots the square of the distance between these two resonances as a function of the square of the modulation frequency $\omega^{\prime}$ one obtains a graph similar to that of Fig. 4 , with $\omega_{1}-\omega_{2}$ replaced by $\omega^{\prime}$.

\section{RESULTS AND DISCUSSION}

The procedure described above has been applied to a number of hydrocarbons. Their choice was motivated by the desire of dealing with simple systems which, at the same time, exhibit some of the more interesting features in their magnetic resonance spectra. The first two molecules are of the simplest imaginable type, containing only two groups with one proton in each group, while the following cases show a stepwise increasing complexity of the spectrum, due to the presence of additional protons and proton groups in the molecule.

The results obtained for various compounds are presented below. The structural formula for each compound, together with photographs of the recorded traces, are shown on the corresponding figures.

\section{A. 2-bromo-5-chlorothiophene: $\mathrm{SCBr}: \mathrm{CHCH}: \mathrm{CCl}$}

The proton resonance of this molecule was first investigated by Hahn and Maxwell ${ }^{3}$ by studying the modulation of the spin-echo. They found that the chemical shift, $\delta$, and the spin coupling, $J$, are in this case of comparable magnitude. This fact is clearly manifested in the spectrum, shown in Fig. 5. The theoretical spectrum for the two proton case is summarized in Table I. Averaging data from five separate recordings the following values were determined for the chemical shift and the spin-spin interaction:

$$
\delta /(2 \pi)=4.7 \pm 0.2 \mathrm{cps}, \quad J /(2 \pi)=3.9 \pm 0.2 \mathrm{cps} .
$$

Using these values of $J$ and $\delta$ the relative line intensities have been calculated yielding the value of the ratio of intensity $R=0.22 \pm 0.01$ of either outer line compared to either inner line. This number agrees to within the experimental error with the corresponding line area ratios which have been measured in a number of recordings yielding an average value of $R=0.195 \pm 0.020$. 


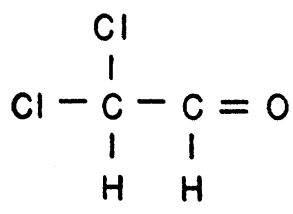

\section{DICHLOROACETALDEHYDE}
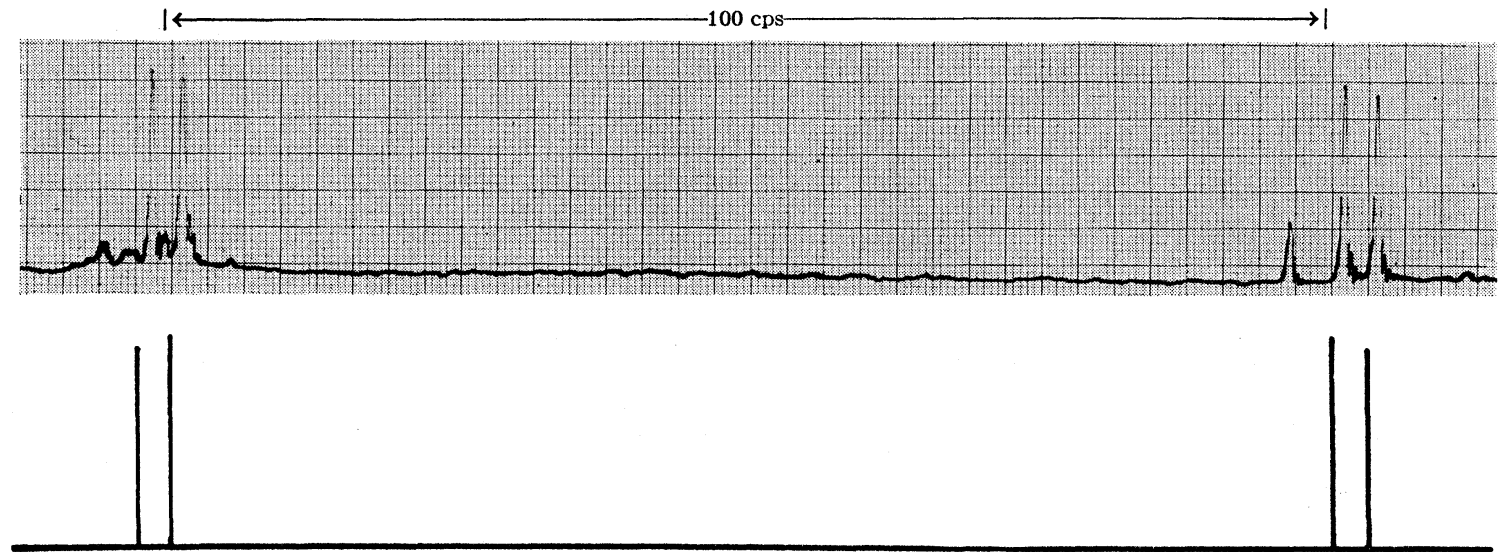

FIG. 6. Spectrum of dichloroacetaldehyde. The small peaks are due to impurities. The theoretical spectrum was calculated assuming that $J / \delta=0.029$.

The results of Hahn and Maxwell corrected for the difference in the external field at which they were obtained, are

$$
\delta /(2 \pi)=5.3 \pm 0.2 \mathrm{cps}, \quad J /(2 \pi)=3.9 \pm 0.2 \mathrm{cps} .
$$

While there is good agreement for the values of $J$ there exists a slight discrepancy of unknown origin for that of $\delta$.

\section{B. Dichloroacetaldehyde, $\mathrm{CHCl}_{2} \mathrm{CHO}$}

Dichloroacetaldehyde, which was likewise previously investigated by Hahn and Maxwell, ${ }^{3}$ has a spectrum shown in Fig. 6. The small peaks are due to impurities which comprise about $10 \%$ of the volume according to the manufacturer's data.

The chemical shift and the spin coupling have been measured and the following values determined:

$$
\delta /(2 \pi)=100.0 \pm 1.0 \mathrm{cps}, \quad J /(2 \pi)=2.9 \pm 0.3 \mathrm{cps} .
$$

They are in good agreement with the values

$$
\delta /(2 \pi)=99.0 \pm 5.0 \mathrm{cps}, \quad J /(2 \pi)=2.7 \pm 0.2 \mathrm{cps},
$$

found by Hahn and Maxwell. ${ }^{3}$

The relative line heights of the two lines on the right hand (low field) side of the trace check with the line intensities predicted by theory, however the impurities present in the sample prevent accurate measurements of the heights of the other two lines.

Since the chemical shift of this two-proton compound is about thirty times larger than the spin coupling, it has been used to demonstrate how the structure, due to spin coupling, disappears in this case by affecting one of the groups with radio-frequency power while ob- serving the other group with a small radio-frequency field. Figure 7 shows a series of traces with the signals arising from transitions produced by the weak radiofrequency field while the strong field was fixed at about $1 \frac{1}{2}$ milligauss, roughly twice the spin-coupling constant when expressed in magnetic field units. The frequency difference between the strong and weak fields was changed between traces. Practically complete disappearance of the structure is observed when the frequency difference is equal to the chemical shift. The width of the trace lines is produced by the vibrations of the spinning sample holder. These vibrations modulate the radio-frequency power picked up by the receiver coil in the head so that a relatively large radio-frequency field produces a correspondingly large alternating voltage in the detector.

\section{1,1,2-trichloroethane, $\mathrm{CHCl}_{2} \mathrm{CH}_{2} \mathrm{Cl}$}

This compound, also investigated by $\mathrm{Hahn}$ and Maxwell, ${ }^{3}$ has the spectrum shown in Fig. 8 and compared with the theoretical results summarized in Table II. From calibration traces the following values were determined for the chemical shift and the spin-spin interaction:

$$
\delta /(2 \pi)=55.5 \pm 1.5 \mathrm{cps}, \quad J /(2 \pi)=5.7 \pm 0.5 \mathrm{cps} .
$$

The results of Hahn and Maxwell are

$$
\delta /(2 \pi)=61.0 \pm 3 \mathrm{cps}, \quad J /(2 \pi)=6.0 \pm 0.3 \mathrm{cps} .
$$

Here the two values of $J$ are just within the estimated error; the values of $\delta$ are just outside the estimated error without, however, representing a significant discrepancy. 


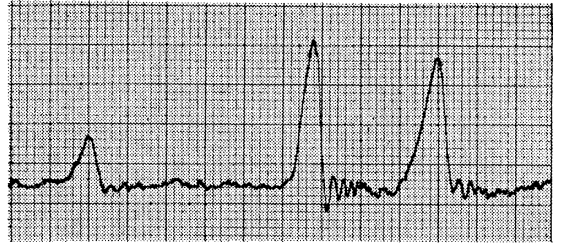

Without radiation

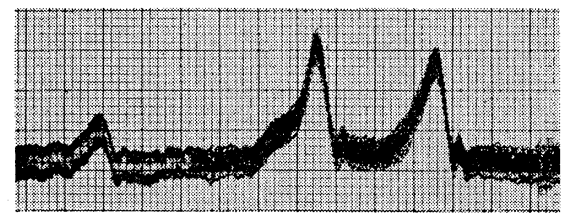

$110 \mathrm{cps}$

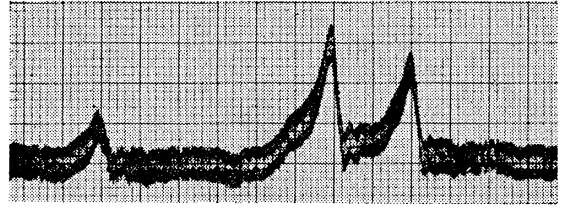

$105 \mathrm{cps}$

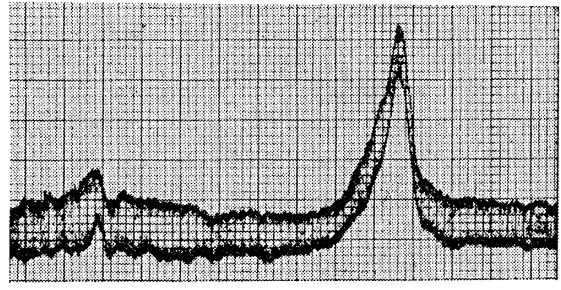

$100 \mathrm{cps}$

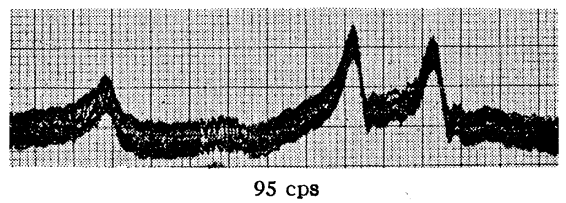

FIG. 7. Resonance in the group appearing at the lower field in dichloroacetaldehyde produced by a small radio-frequency field of angular frequency $\omega_{2}$, while a strong radio-frequency field of angular frequency $\omega_{1}$ is applied near the resonance frequency of the other group. The amplitude of the strong field is about $1 \frac{1}{2}$ milligauss. The frequency difference of the two fields $\left(\omega_{2}-\omega_{1}\right) /(2 \pi)$ is given below each trace. The small peak on the left of each trace is the result of an impurity, and it may be used as a reference mark.

This compound has two groups of protons with two equivalent protons in one group, $B$, and one proton in the other group, $A$. The two peaks on the left-hand side in Fig. 8(A) are associated with transitions of the two equivalent protons in group $B$, the two lines arising from the two possible states states $m_{A}= \pm \frac{1}{2}$. According to theory, each of these lines should be doubled as is explained in Sec. II; however this splitting amounts to less than $0.4 \mathrm{cps}$ and is not resolved.

The group of lines at the right-hand side of Fig. 8 is associated with transitions in the group $A$, the three main lines arising from the three possible values of $m_{B}= \pm 1$ or 0 . The central line is double, arising from the fact that $m_{B}=0$ can be obtained both for the case total spin $I_{B}=1$ and $I_{B}=0$, and that the energy values depend not only on $m_{B}$ but also upon $I_{B}$.

It is of interest to note that while the relative line height of the three lines associated with $I_{B}=1$ agree with the predicted line intensity, the line associated with $I_{B}=0$ is $50 \%$ higher than the theoretical line intensity. It is reasonable to suppose that this increase in height is accompanied by a proportional decrease in width, which is seen in a qualitative way in Fig. $8(\mathrm{C})$. The width of this line cannot be accurately measured since the two lines for $m_{B}=0$ are not completely resolved; however, the combined area of the two lines has been measured and found to be in agreement with the theoretical relative intensity.

The observation of different line widths may be understood in a qualitative way by noting that the widths of lines in one group will be affected by the lifetimes of states of the other group. ${ }^{14}$ Applying this consideration to this case one finds that the state associated with $I_{B}=0, m_{B}=0$ is relatively long lived compared to the states associated with $I_{B}=1, m_{B}=0$ or \pm 1 . This fact indicates that changes of the total spin $I_{B}$ are relatively infrequent, a circumstance which can be explained by assuming that the molecular surroundings are very nearly symmetrical in the two protons of this group.

\section{Q-propiolactone, $\mathrm{CH}_{2} \mathrm{CH}_{2} \mathrm{COO}$}

This compound has the spectrum shown in Fig. 9. This spectrum is in agreement with the assumption that there are two groups of equivalent protons with two protons in each group. With this assumption the theoretical line spacings and intensities have been calculated for the ratio $J / \delta=0.265$ and is compared with experimental values in Table III.

TABLE III. Relative transition frequencies and line intensities for the case of two groups with two protons in each group for the case of $J / \delta=0.265$ are calculated and compared with measured values taken from the trace of $\beta$-propiolactone shown in Fig. 9. Only relative transition frequencies for group $A$ are listed; those of group $B$ are of equal magnitude but of opposite sign to those of group $A$.

\begin{tabular}{|c|c|c|c|c|c|}
\hline \multirow{3}{*}{\multicolumn{2}{|c|}{$\begin{array}{l}\text { Transition in } \\
\text { limit of } J \rightarrow 0 \\
m_{A} \longleftrightarrow m_{A}-1\end{array}$}} & \multicolumn{2}{|c|}{ Calculated values } & \multicolumn{2}{|c|}{ Measured values } \\
\hline & & \multirow{2}{*}{$\begin{array}{l}\text { frequency } \\
\text { in units } \\
\text { of } \delta\end{array}$} & \multirow{2}{*}{$\begin{array}{l}\text { Relative } \\
\text { intensity }\end{array}$} & \multirow{2}{*}{$\begin{array}{c}\text { Relative } \\
\text { line } \\
\text { spacing }\end{array}$} & \multirow{2}{*}{$\begin{array}{c}\text { Relative } \\
\text { line } \\
\text { height }\end{array}$} \\
\hline & & & & & \\
\hline 1 & 1 & 08319 & 1.06 & 0.832 & 11 \\
\hline 0 & 1 & 0.7528 & 1.27 & 0.756 & 1.3 \\
\hline 1 & 0 & 0.6011 & 1.48 & 0.596 & 1.4 \\
\hline 0 & 0 & 0.5327 & 1.78 & 0.526 & 2.7 \\
\hline 1 & $\left.\begin{array}{l}0^{2} \\
0^{a}\end{array}\right\}$ & 0.5000 & 4.00 & 0.500 & 4.8 \\
\hline $\begin{array}{l}0 \\
0\end{array}$ & $\begin{array}{c}\left.0^{\mathrm{a}}\right) \\
-1\end{array}$ & 0.3019 & 2.93 & 0.302 & 2.9 \\
\hline 1 & -1 & 0.2570 & 3.44 & 0.255 & 3.1 \\
\hline
\end{tabular}

a Transition with $I_{A}=1, I_{B}=0$; all other transitions with $I_{A}=1, I_{B}=1$.

${ }^{14} \mathrm{~F}$. Bloch, this issue [Phys. Rev. 102, 104 (1956)], Sec. 6. 
<smiles>ClCC(Cl)Cl</smiles>

\section{1,1,2-TRICHLOROETHANE}

(A)

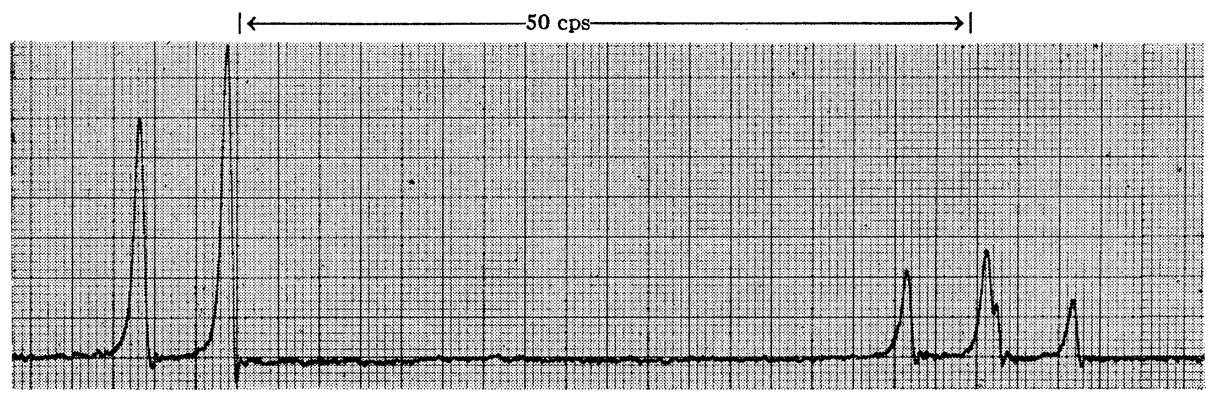

(B)
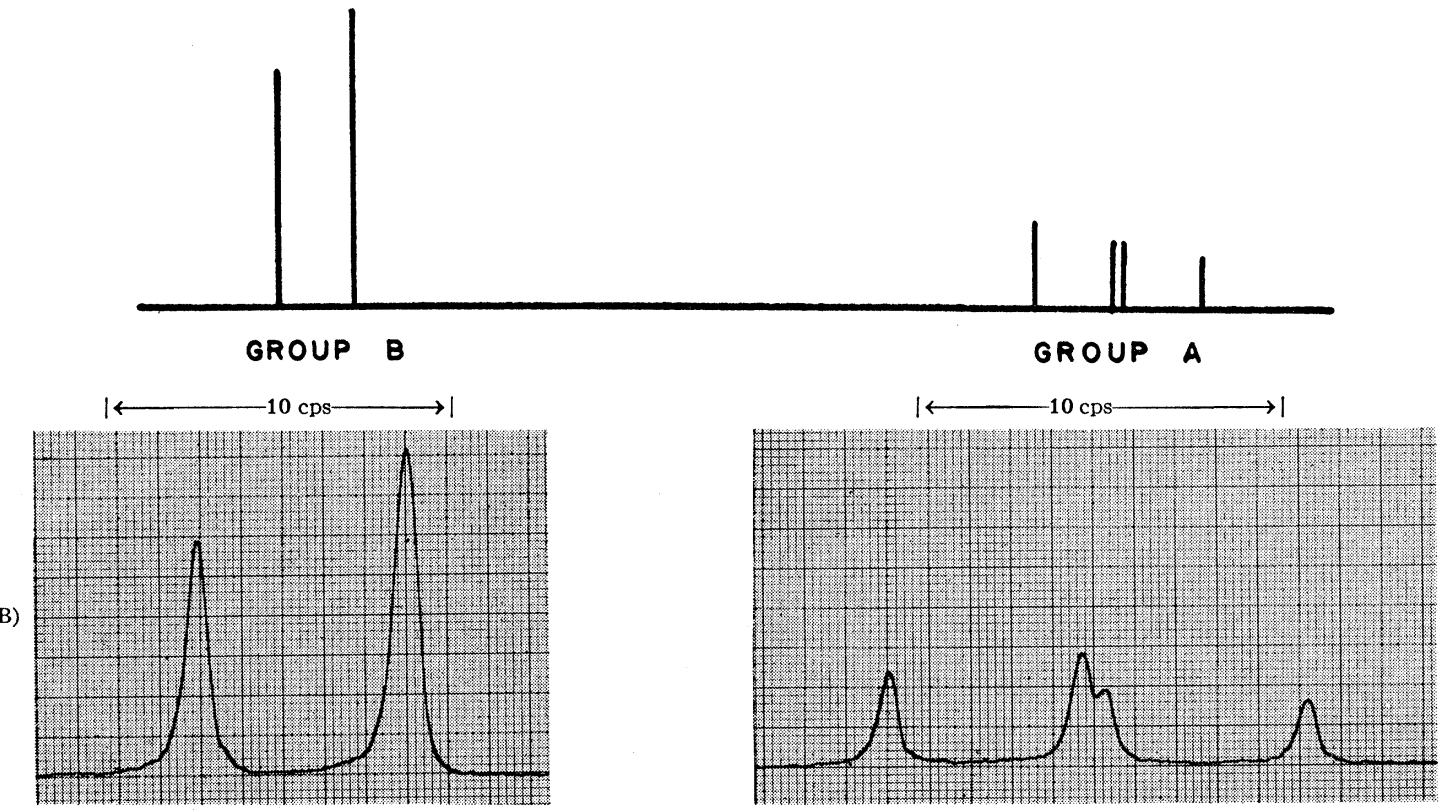

(C)

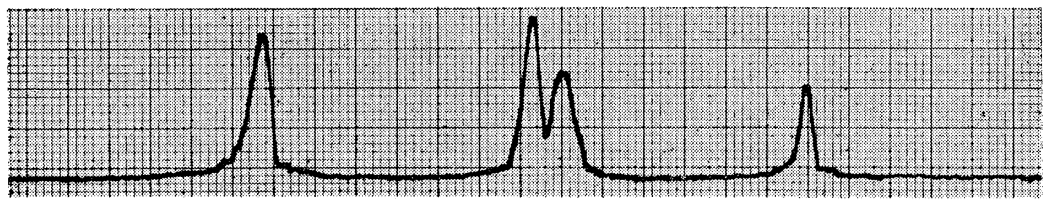

FIG. 8. Spectrum of 1,1,2-trichloroethane. (A) Total spectrum with theoretical line spectrum below. The theoretical spectrum was derived from the expression given in Table II assuming $J / \delta=0.105$. The lines occuring at the higher frequency appear at the right-hand side of the trace. Although each of the two lines in group $B$ should be doubled, the splitting is too small to be resolved. Single lines are therefore drawn in the theoretical intensity pattern each with a length equal to the sum of the lengths of the two lines which they represent. (B) The spectrum of each group obtained by sweeping the magnetic field more slowly. (C) The spectrum of group $A$ under better resolution; however, the right-hand peak appears somewhat distorted, probably because of nonlinearities in the rate of sweep of the magnetic field. In this trace one of the two central lines is associated with the state $I_{B}=0, m_{B}=0$ of group $B$ and it definitely appears narrower as well as higher than the adjacent other line, associated with the state $I_{B}=1$, $m_{B}=0$.

The extremely good agreement would suggest that the above assumption of the protons within each group being equivalent is justified. It is generally believed that this molecule has a plane of symmetry with the carbon and oxygen atoms lying in this plane and the protons placed symmetrically above and below the plane. If there is no exchange of protons there would be no a priori reason to expect that a proton in one group would have the same spin-coupling constant to each of the protons in the other group. The calculation of 


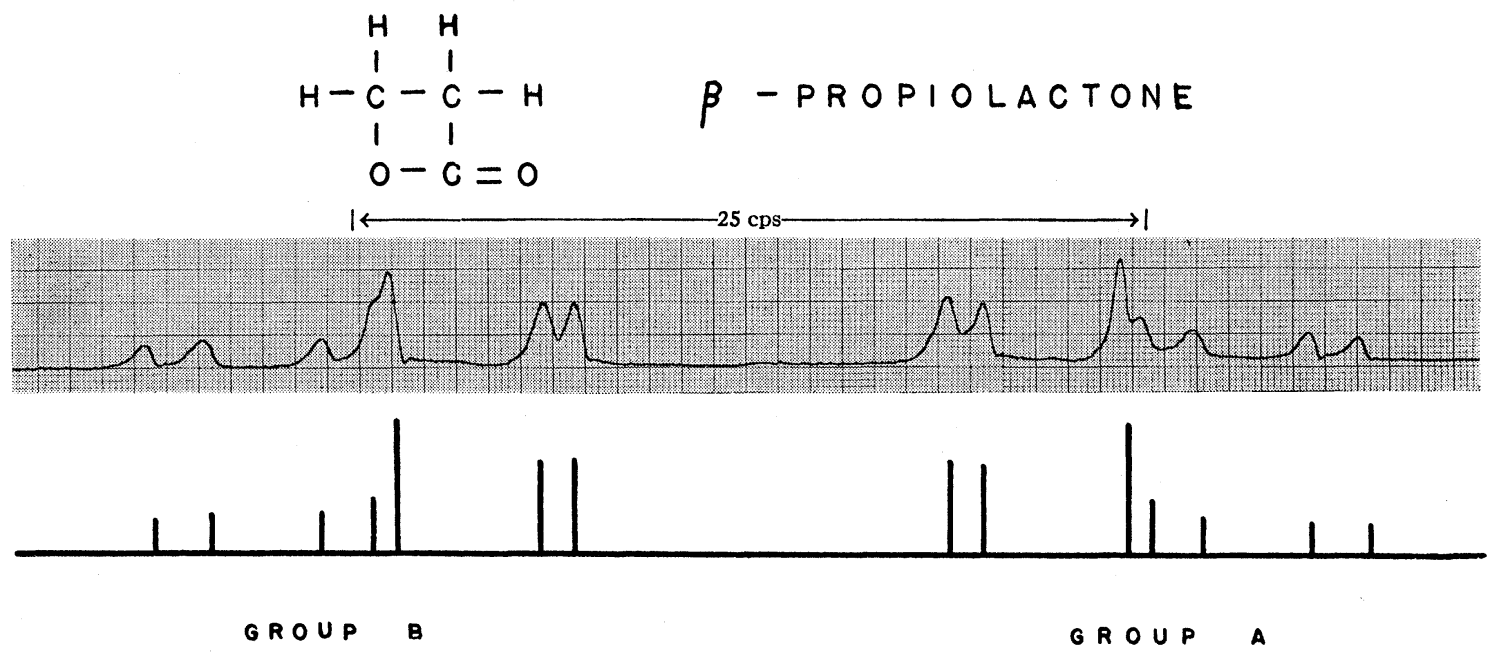

FIG. 9. Spectrum of $\beta$-propiolactone. The theoretical line spectrum below was calculated in the manner outlined in Sec. II for the case of $J / \delta=0.265$ with the results given in Table III.

the energy levels in this more general case is more complicated since the protons in each group are not equivalent and thus the total spin of a group is not a good quantum number. However, since there is a plane of symmetry it is convenient to choose initial wavefunctions which preserve this symmetry. ${ }^{15}$ These calculations show that there would be additional lines in the spectrum if the protons in each group were not closely equivalent.

The absolute value of the chemical shift and the spin coupling have been determined and found to be

$$
\delta /(2 \pi)=23.0 \pm 1.0 \mathrm{cps}, \quad J /(2 \pi)=6.1 \pm 0.3 \mathrm{cps} .
$$

\section{E. 2,3-dibromopropene, $\mathrm{CH}_{2}: \mathrm{CBrCH}_{2} \mathrm{Br}$}

The spectrum of this compound, shown in Fig. 10 shows that there are three groups of nonequivalent protons. The protons bound to the double-bonded carbon atom are nonequivalent because the double bond prevents free rotation of this group, so that one proton is always on the same side of the double bond as the bromine atom (cis) and the other proton is across from the bromine atom (trans).

The two protons associated with the $\mathrm{CH}_{2} \mathrm{Br}$ group are equivalent. Transitions of protons within this group appear to the left (higher-field side) of the trace in Fig. 10 (A) as may be deduced by the fact that the total line intensity of this multiplet is about twice that of either of the two other multiplets. It is not possible to determine from the spectrum which of the other two multiplets is associated with the trans group and which with the cis group. The fact that the furthest line to the left side of the trace is higher than the adjacent line contrary to what is expected theoretically is believed to be due to impurities within the sample.

${ }_{15}$ Problems similar to this one are treated by McConnell, McLean, and Reilly, J. Chem. Phys. 23, 1152 (1955).
In order to associate beyond doubt the fine structure on the right-hand multiplet of Fig. 10(A) with a definite spin coupling, a double irradiation experiment was performed. When the $\mathrm{CH}_{2} \mathrm{Br}$ group was irradiated with a large radio-frequency field while observing the righthand multiplet with a small field, the five-line pattern in Fig. 10(B) reduced to two lines, as shown in Fig. 10(C). This residual structure must be due to the spin coupling between the two single protons and shows that the higher multiplicity, shown in Fig. 10(B), has to be ascribed to the other proton group. From calibration traces the values of the various chemical shifts and spin couplings have been determined. The groups are denoted by letters $A, B, C$ in the order of increasing values of their resonance fields; the resonance of group $A$ occurs at the lowest field [the right-hand side of the trace in Fig. 10(A)], and the shifts are denoted by $\delta_{R S}=\omega_{R}-\omega_{S}$.

$$
\begin{array}{ll}
\delta_{A B} /(2 \pi)=13.0 \pm 1.0 \mathrm{cps}, & J_{A B} /(2 \pi)=2.2 \pm 0.3 \mathrm{cps}, \\
\delta_{A C} /(2 \pi)=56.0 \pm 1.0 \mathrm{cps}, & J_{A C} /(2 \pi)=1.0 \pm 0.2 \mathrm{cps}, \\
\delta_{B C} /(2 \pi)=43.0 \pm 1.0 \mathrm{cps}, & J_{B C} /(2 \pi)<0.3 \mathrm{cps} .
\end{array}
$$

We have not been able to determine the relative signs of the above spin couplings. In order to determine these signs one must have sufficient resolution to see the effects which are given by second-order perturbation theory.

The theoretical line intensities using first-order perturbation theory are compared to the measured intensities in Table IV.

\section{F. Ethyl bromide, $\mathrm{CH}_{3} \mathrm{CH}_{2} \mathrm{Br}$}

This compound has the spectrum shown in Fig. 11. With two protons in one group and three in the other this spectrum has an appearance very similar to the corresponding groups of ethyl alcohol and yields a number of closely spaced resonance lines. 


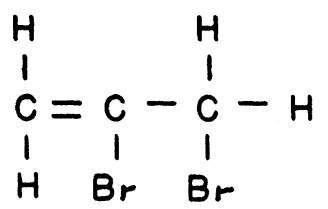

\section{$2,3-D I B R O M O P R O P E N E$}
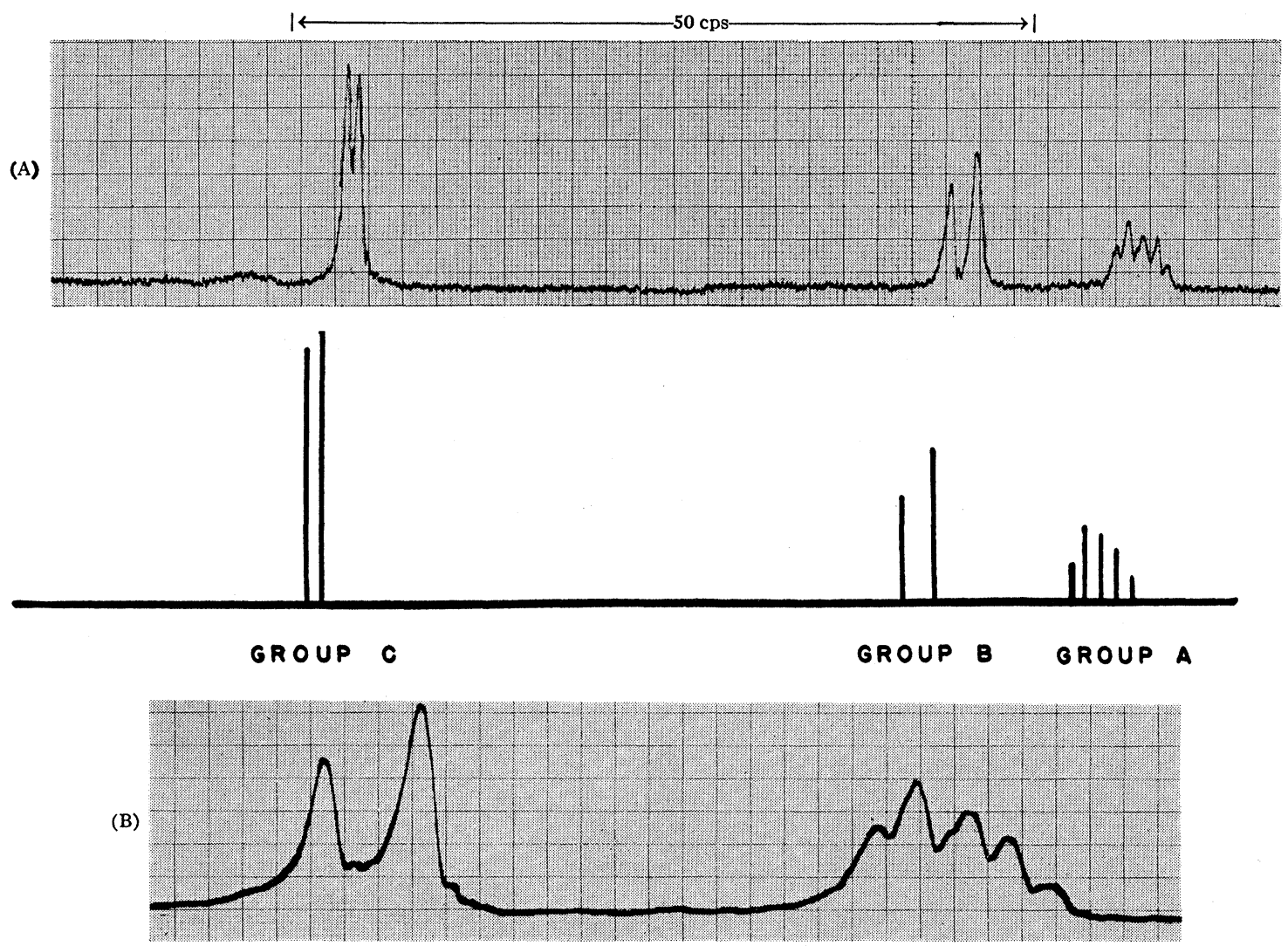

(C)

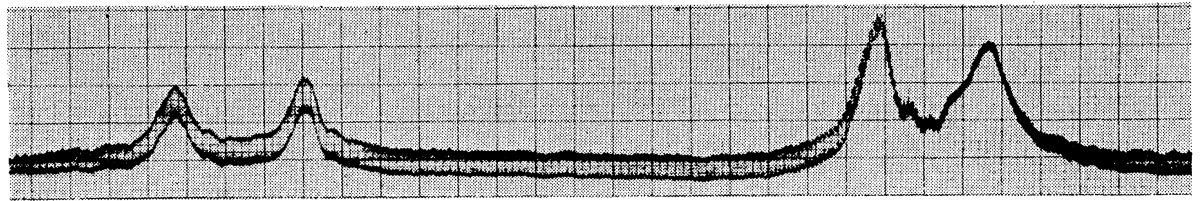

FIG. 10. Spectrum of 2,3-dibromopropene. (A) Total spectrum with the theoretical line spectrum below. The theoretical spectrum was derived using the first-order perturbation theory developed in Appendix II with the results listed in Table IV. The transitions which occur at the higher frequency appear at the right-hand side of the trace. (B) Spectrum of groups $A$ and $B$ made just before group $C$ was irradiated with a large radio-frequency field. (C) Spectrum of groups $A$ and $B$ investigated with a small radio-frequency field while a large radio-frequency field was applied at a frequency which was lower by 56 cycles per second. Thus while group $A$ was in resonance, group $C$ was being strongly affected by radio-frequency power.

Labeling the group with 2 protons by the letter $A$ and the group with 3 protons by the letter $B$, we note that the spin $I_{A}$ may take the values of 1 or 0 and the spin $I_{B}$ the values $\frac{3}{2}$ or $\frac{1}{2}$, the latter state having a statistical weight of 2 . Since the spins of each group are good quantum numbers the problem of solving the energy levels, transition frequencies and line intensities may be broken into three parts: (1) $I_{A}=0, I_{B}=\frac{3}{2}$; (2) $I_{A}=1$ or $0, I_{B}=\frac{1}{2}$; (3) $I_{A}=1, I_{B}=\frac{3}{2}$. The first part is trivial yielding a single transition frequency $\omega_{B}$. The second part is identical to the problem with 2 prot on in one group and one in the other which is discussed above with the results given in Table II. The solution to the third part involves two cubic equations which have been solved numerically for the ratio $J / \delta=0.136$. The results of all three parts of the calculation are summarized in Table $\mathrm{V}$ and compared with the observed transition frequencies and line heights.

From measurements of the line spacings the values of the chemical shift and the spin coupling have been 
TABLE IV. Using the expressions derived in Appendix II in first order, the transition frequencies and line intensities are calculated for 2,3-dibromopropene. They are compared to the measured line heights, the data being taken from the trace in Fig. 10(A).

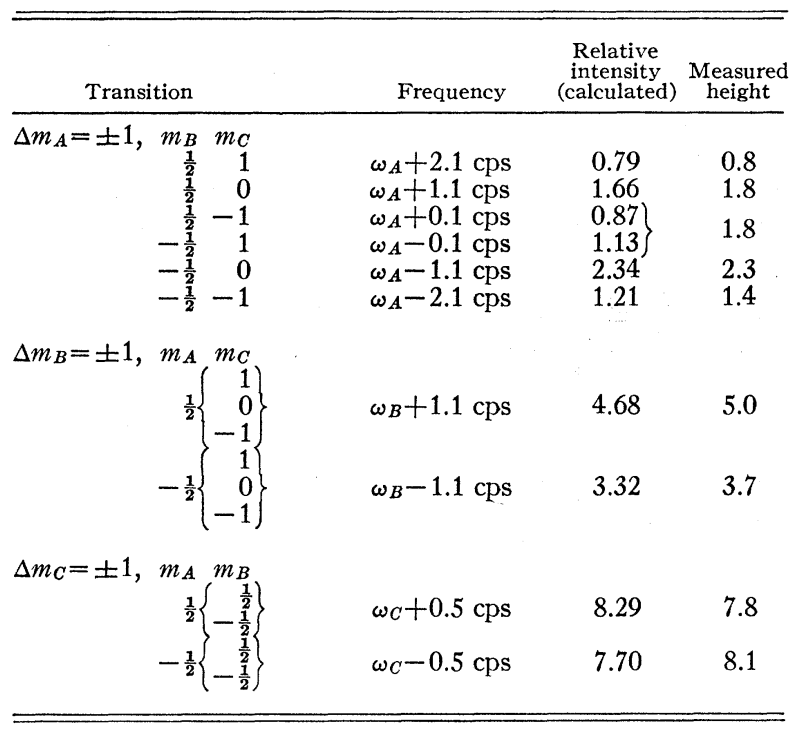

determined and found to be

$$
\delta /(2 \pi)=52.7 \pm 1.5 \mathrm{cps}, \quad J /(2 \pi)=7.2 \pm 0.5 \mathrm{cps} .
$$

In this molecule there is also evidence of different linewidths. Perhaps these differences are most easily detected by observing the transient oscillations ${ }^{16}$ which occur after passing through some of the resonances and
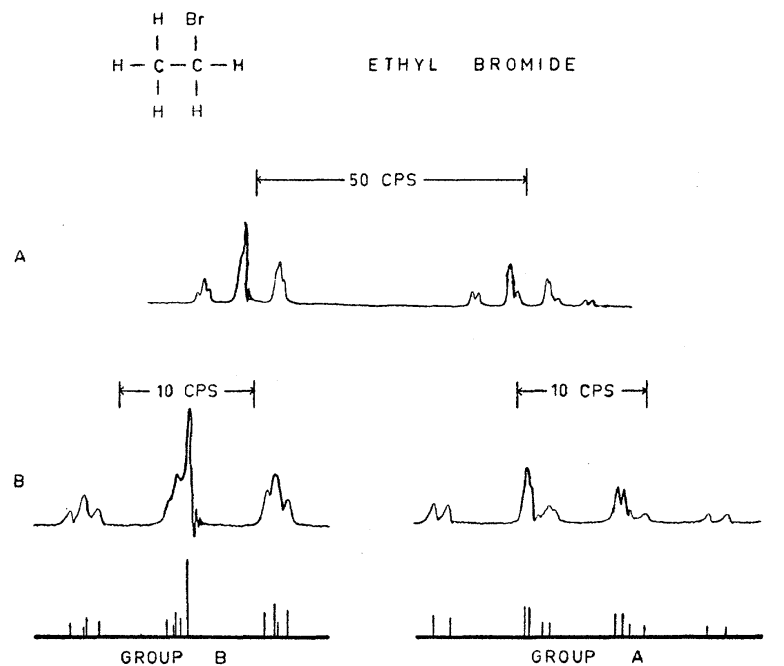

Fig. 11. The spectrum of ethyl bromide. (A) The entire spectrum. The transitions which occur at the higher frequency appear at the right-hand side of the trace. (B) The spectrum of each group obtained by sweeping the magnetic field more slowly. The theoretical line spectrum drawn below was calculated in the manner outlined in Sec. II for a ratio $J / \delta=0.136$ with the results given in Table $\mathrm{V}$.

${ }^{16}$ B. A. Jacobsohn and R. K. Wangsness, Phys. Rev. 73, 942 (1948). not others. The most pronounced transient occurs after the transition at frequency $\omega_{B}$ corresponding to the state $I_{A}=0$. This indicates that this line is narrower and thus should be correspondingly higher which may be seen by the comparison of line heights in Table V(A). Small transients also appear after the transitions in group $A$ corresponding to $I_{B}=\frac{1}{2}$, indicating that these lines are narrower than the lines with $I_{B}=\frac{3}{2}$. Unfortunately at present the resolution is not sufficiently high to permit any quantitative measurements.

\section{CONCLUSIONS}

It has been demonstrated that the many fine features of the proton spectra of hydrocarbons which we have observed with increasingly high resolution may be explained by a relatively simple Hamiltonian. Although the compounds studied were of known structure the results obtained here may serve as a useful guide for nuclear magnetic studies of other and more complicated molecules. The occurrence of different line widths in the same spectrum appears to offer additional information about relaxation mechanisms and it would appear to justify further efforts to improve the resolution until

TABLE $V$. The transition frequencies and line heights of the ethyl bromide spectrum in Fig. 11 are compared to a calculated spectrum with two protons in group $A$ and three protons in group $B$ and the ratio $J / \delta=0.136$.

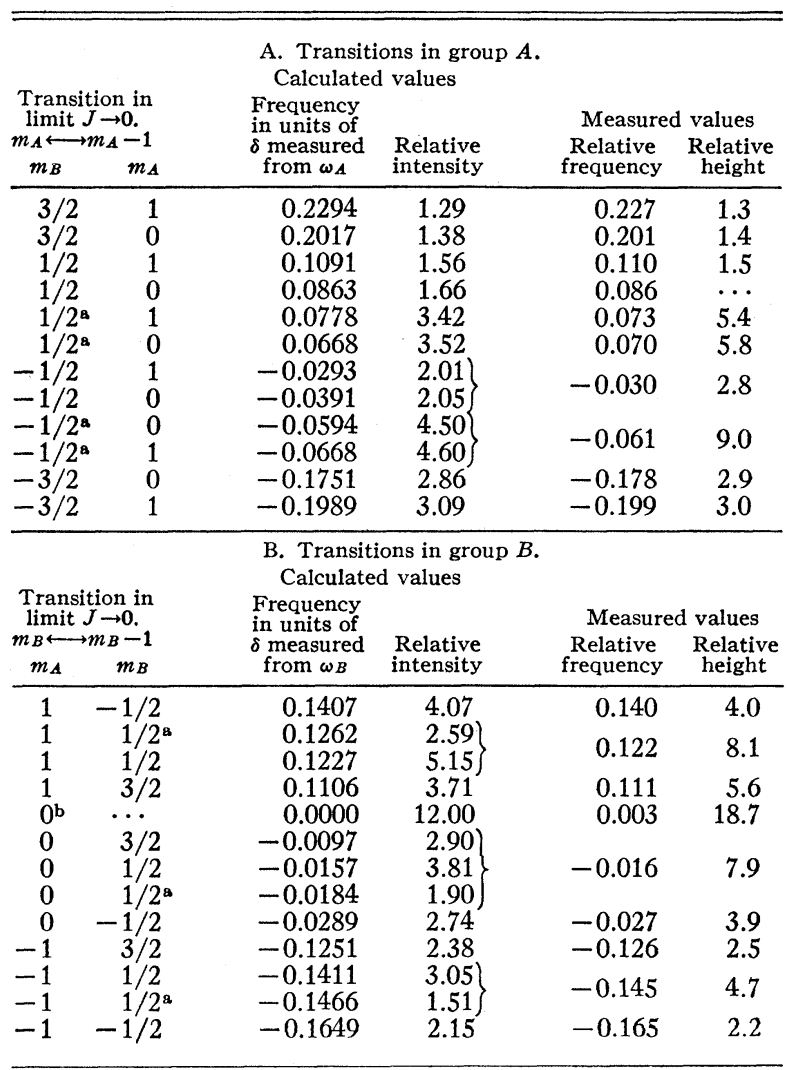

a Transitions with $I_{B}=1 / 2$; others with $I_{B}=3 / 2$ b All transitions of group $B$ with $I_{A}=0$. 
one observes the natural line width rather than that determined by the inhomogenities of the applied field.

\section{ACKNOWLEDGMENTS}

The author wishes to express his gratitude to the many individuals who have contributed to this research. In particular he wishes to thank Professor Felix Bloch for his friendly interest and much helpful advice, and Dr. J. T. Arnold and Dr. S. Meiboom for their assistance and collaboration.

\section{APPENDIX I. DERIVATION OF RESONANCE CONDI- TION WHEN TWO SIMULTANEOUS RADIO-FRE- \\ QUENCY FIELDS ARE APPLIED TO A GROUP OF UNCOUPLED NUCLEI OF SPIN $1 / 2$}

Consider the phenomenological equations ${ }^{17}$ with $m=M_{x}+i M_{y}, h=\gamma\left(H_{x}+i H_{y}\right)$, and $\omega_{A}=\gamma H_{z}$. The equations are then

$$
\begin{aligned}
\dot{m}+i \omega_{A} m-i h M_{z}+m / T_{2} & =0, \\
\dot{M}_{z}+\operatorname{Im}\left(m h^{*}\right)+M_{z} / T_{1} & =M_{0} / T_{1},
\end{aligned}
$$

where Im stands for the imaginary part, and ${ }^{*}$ for the complex conjugate. Let

$$
\begin{aligned}
h=h_{1} \exp \left(-i \omega_{1} t\right)+h_{2} & \exp \left(-i \omega_{2} t\right) \\
= & \left\{h_{1}+h_{2} \exp \left(-i \omega^{\prime} t\right)\right\} \exp \left(-i \omega_{1} t\right),
\end{aligned}
$$

where $\omega^{\prime}=\omega_{2}-\omega_{1}$. Assume $h_{1} \gg h_{2}$ and $h_{1}, h_{2}$ to be real, and further let

$$
m=\left\{m_{1}+m_{2}(t)\right\} \exp \left(-i \omega_{1} t\right), \quad M_{z}=M_{1}+M_{2}(t),
$$

where $m_{1}, M_{1}$ are constant in time and $m_{2}, M_{2}$ are small perturbations. The terms with subscript 1 are of zero order in the perturbation, and those with subscript 2 are of first order. The zero-order equations are

$$
\begin{aligned}
i \Delta \omega m_{1}-i h_{1} M_{1}+m_{1} / T_{2} & =0, \\
\operatorname{Im}\left(m_{1} h_{1}\right)+M_{1} / T_{1} & =M_{0} / T_{1},
\end{aligned}
$$

where $\Delta \omega=\omega_{A}-\omega_{1}$. These equations yield the usual slow passage solutions to the phenomenological equations. The first-order perturbation equations are

$$
\begin{aligned}
& \dot{m}_{2}+i \Delta \omega m_{2}-i M_{1} h_{2} \exp \left(-i \omega^{\prime} t\right)-i M_{2} h_{1}+m_{2} / T_{2}=0, \\
& \dot{M}_{2}+\operatorname{Im}\left\{m_{1} h_{2} \exp \left(i \omega^{\prime} t\right)+m_{2} h_{1}\right\}+M_{2} / T_{1}=0 .
\end{aligned}
$$

A solution of the following form is assumed:

$$
\begin{aligned}
& m_{2}=A \exp \left(-i \omega^{\prime} t\right)+B^{*} \exp \left(i \omega^{\prime} t\right), \\
& M_{2}=C \exp \left(-i \omega^{\prime} t\right)+C^{*} \exp \left(i \omega^{\prime} t\right) .
\end{aligned}
$$

Upon substituting the assumed values into the differential equations and equating coefficients of the exponentials one finds three complex equations for the coefficients $A, B$, and $C$. These equations expressed in

\footnotetext{
${ }^{17}$ F. Bloch, Phys. Rev. 70, 460 (1946).
}

matrix notation have the following form:

$$
\begin{array}{rcc}
\left(\begin{array}{ccc}
1+i\left(\Delta \omega-\omega^{\prime}\right) T_{2} & 0 & -i h_{1} T_{2} \\
0 & 1-i\left(\Delta \omega+\omega^{\prime}\right) T_{2} & i h_{1} T_{2} \\
-\frac{1}{2} i h_{1} T_{1} & \frac{1}{2} i h_{1} T_{1} & 1-i \omega^{\prime} T_{1}
\end{array}\right]\left(\begin{array}{l}
A \\
B \\
C
\end{array}\right) & =\left[\begin{array}{c}
i M_{1} h_{2} T_{2} \\
0 \\
-\frac{1}{2} i m_{1} * h_{2} T_{1}
\end{array}\right)
\end{array}
$$

The solution for the constant $A$ may be written as the quotient of two determinants

$$
A=\frac{\left|\begin{array}{ccc}
i h_{2} T_{2} M_{1} & 0 & -i h_{1} T_{2} \\
0 & 1-i\left(\Delta \omega+\omega^{\prime}\right) T_{2} & i h_{1} T_{2} \\
-\frac{1}{2} i h_{2} T_{1} m_{1} * & \frac{1}{2} i h_{1} T_{1} & 1-i \omega^{\prime} T_{1}
\end{array}\right|}{\left|\begin{array}{ccc}
1+i\left(\Delta \omega-\omega^{\prime}\right) T_{2} & 0 & -i h_{1} T_{2} \\
0 & 1-i\left(\Delta \omega+\omega^{\prime}\right) T_{2} & i h_{1} T_{2} \\
-\frac{1}{2} i h_{1} T_{1} & \frac{1}{2} i h_{1} T_{1} & 1-i \omega^{\prime} T_{2}
\end{array}\right|}
$$

Resonance will occur when the determinant in the denominator has its minimum value, provided that the determinant in the numerator is not at the same time vanishingly small. For long relaxation times we need to keep only the terms with the highest powers of $T_{1}$ and $T_{2}$. The determinant, $D$, of the denominator then has the approximate value

$$
\begin{aligned}
D=i \omega^{\prime} T_{1} T_{2}{ }^{2}\left(\omega^{\prime 2}-\Delta \omega^{2}-h_{1}^{2}\right)+ & T_{1} T_{2}\left(h_{1}{ }^{2}-2 \omega^{\prime 2}\right) \\
& +T_{2}^{2}\left(\Delta \omega^{2}-\omega^{\prime 2}\right)+\cdots .
\end{aligned}
$$

Resonance occurs when the coefficient of the highest power of $T_{1}, T_{2}$ vanishes, i.e., when $\left(\Delta \omega^{2}\right)=\omega^{2}-h_{1}^{2}$. With $\Delta \omega=\omega_{A}-\omega_{1}, h_{1}=\gamma H_{1}$, and $\omega^{\prime}=\omega_{2}-\omega_{1}$, the resonance condition is

$$
\left(\omega_{2}-\omega_{2}\right)^{2}=\left(\omega_{A}-\omega_{1}\right)^{2}+\gamma^{2} H_{1}^{2} .
$$

as stated in (4) in Sec. I.

The value of $A$ at resonance has been determined using the same approximation of long relaxation times.

$$
A_{0}=\frac{ \pm i h_{2} T_{1} T_{2}^{2} M_{0} \cos \theta(1 \pm \cos \theta)^{2}}{2\left\{T_{1}\left(1+\cos ^{2} \theta\right)+T_{2} \sin ^{2} \theta\right\}\left\{T_{2} \cos ^{2} \theta+T_{1} \sin ^{2} \theta\right\}},
$$

where $A_{0}$ denotes the value of $A$ at resonance and $\sin \theta=h_{1} / \omega^{\prime}$. The upper sign is taken when $\omega^{\prime}$ and $\Delta \omega$ have like signs and the lower sign taken when they have unlike signs. It is seen in the limit $\omega^{\prime} \gg h_{1}$ that $\theta \rightarrow 0$ and one resonance becomes vanishingly small while the other resonance fulfills the usual resonance condition $\omega_{2} \rightarrow \omega_{A}$ with the usual resonance amplitude $+i h_{2} T_{2} M_{0}$.

The height of the signal produced by the weak radiofrequency field is proportional to $A$ and the shape of the signal near resonance is thus given by

$$
A=A_{0} \Gamma /(\Gamma+i \epsilon),
$$

where $\Gamma$ is the line-width parameter and $\epsilon$ is the deviation from the responance expressed in units of angular frequency. If resonance is produced by sweeping the 
frequency $\omega_{2}$ of the weak radio-frequency field, then $\epsilon$ is given by

$$
\epsilon=\omega^{\prime} \pm\left\{\Delta \omega^{2}+h_{1}^{2}\right\}^{\frac{1}{2}},
$$

and the line-width parameter $\Gamma$ given by

$$
\Gamma=1 / T_{2}-\frac{1}{2}\left(1 / T_{2}-1 / T_{1}\right) \sin ^{2} \theta .
$$

This result agrees with that of Eq. (7.96) in the accompanying paper by Bloch, where it was derived in a somewhat different manner. However in the experiments considered in this paper the magnetic field was swept through resonance and thus the observed line width $\Gamma^{\prime}$ is not that given by the equation above. For this case the deviation from resonance may be expressed by

$$
\epsilon^{\prime}=\Delta \omega \pm\left\{\omega^{\prime 2}-h_{1}^{2}\right\}^{\frac{1}{2}},
$$

and the observed line width $\Gamma^{\prime}$ has been calculated and found to be given by the following expression:

$$
\Gamma^{\prime}=\left\{1 / T_{2}-\frac{1}{2}\left(1 / T_{2}-1 / T_{1}\right) \sin ^{2} \theta\right\} / \cos \theta .
$$

It may be noted that as the two resonances approach each other the observed line width becomes greater, a fact which may be observed in a qualitative way in Fig. 3.

\section{APPENDIX II. PERTURBATION CALCULATION OF THE ENERGY LEVELS AND LINE INTENSITIES FOR AN ARBITRARY NUMBER OF NUCLEAR GROUPS BUT WITH ONLY EQUIVA- LENT NUCLEI IN EACH GROUP}

The unperturbed Hamiltonian is that of an arbitrary number of uncoupled groups,

$$
\mathfrak{H}^{(0)}=-\sum_{R} \omega_{R} M_{R},
$$

with a total energy of

$$
E^{(0)}\left(m_{A}, m_{B}, \cdots\right)=-\sum_{R} \omega_{R} m_{R} .
$$

We assume the spin coupling

$$
\mathcal{H}^{(1)}=-\frac{1}{2} \sum_{R} \sum_{S \neq R} J_{R S}\left\{M_{R} M_{S}+M_{R}{ }^{+} M_{S^{-}}\right\}
$$

is a small perturbation. The first order correction to the energy levels are the diagonal matrix elements of the perturbation

$$
E^{(1)}\left(m_{A}, m_{B}, \cdots\right)=-\frac{1}{2} \sum_{R} \sum_{S \neq R} J_{R S} m_{R} m_{S} .
$$

The second-order perturbation correction to the energy is

$$
E^{(2)}(i)=\sum_{j \neq i} \frac{\left(i\left|\mathcal{F}^{(1)}\right| j\right)\left(j\left|\mathfrak{F}^{(1)}\right| i\right)}{E^{(0)}(i)-E^{(0)}(j)}
$$

where the indices $i, j$ refer to all of the quantum numbers $I_{A}, I_{B}, \cdots, m_{A}, m_{B}, \cdots$, of the unperturbed system. The nondiagonal matrix elements $\left(i\left|\mathcal{F}^{(1)}\right| j\right)$ are identical with those of the total Hamiltonian and are given by (10) of Sec. II. Upon substituting the values for the matrix elements, one obtains after simplifying:

$$
\begin{aligned}
E^{(2)}\left(m_{A}, m_{B}, \cdots\right)=-\frac{1}{4} \sum_{\substack{R=A, B \\
S \neq R}} \frac{J_{R S^{2}}}{\left(\omega_{R}-\omega_{S}\right)} \\
\quad \times\left\{m_{R}\left(I_{S}{ }^{2}+I_{S}-m_{S}{ }^{2}\right)-m_{S}\left(I_{R}{ }^{2}+I_{R}-m_{R}^{2}\right)\right\} .
\end{aligned}
$$

Thus for the case of only two groups one obtains (14) of Sec. II.

Including second-order terms the frequency of a transition between a state with energy $E\left(m_{A}, m_{B}, \cdots\right)$ and another with energy $E\left(m_{A}-1, m_{B}, \cdots\right)$ is

$$
\begin{aligned}
\omega=\omega_{A}+\sum_{R \neq A} J_{A R} m_{R}+\frac{1}{2} \sum_{R \neq A} \frac{J_{A R^{2}}}{\left(\omega_{A}-\omega_{R}\right)} \\
\times\left\{I_{R}\left(I_{R}+1\right)-m_{R}\left(m_{R}+1\right)+2 m_{A} m_{R}\right\} .
\end{aligned}
$$

Small deviations in some of the lines were found upon comparing the frequencies predicted by this equation to those found in the spectrum of ethyl alcohol. ${ }^{1}$ To determine if these deviations arose from higher order terms, the energy correction in third order was calculated.

The third-order perturbation correction to the energy levels is

$$
\begin{aligned}
E^{(3)}(i)=\sum_{j \neq i} \sum_{k \neq i} \frac{\left(i\left|\mathcal{F}^{(1)}\right| j\right)\left\{\left(j\left|\mathcal{F H}^{(1)}\right| k\right)-E^{(1)} \delta_{j k}\right\}}{E^{(0)}(i)-E^{(0)}(j)} & \\
& \times \frac{\left(k\left|\mathcal{H}^{(1)}\right| i\right)}{E^{(0)}(i)-E^{(0)}(k)} .
\end{aligned}
$$

Upon substituting the values of the matrix elements and simplifying, one obtains

$$
\begin{aligned}
& E^{(3)}\left(m_{A}, m_{B}, \cdots\right)=\sum_{R} \sum_{S \neq R} \frac{J_{R S^{3}} F(R)}{2\left(\omega_{R}-\omega_{S}\right)^{2}} \\
& \times\left\{\frac{1}{2} F(S)+m_{S}\left(1-m_{S}+m_{R}\right)\right\} \\
& +\sum_{R} \sum_{S \neq R} \sum_{T \neq R, S} \frac{J_{R S^{2} J_{R T} m_{T}}}{2\left(\omega_{R}-\omega_{S}\right)^{2}}\left\{F(S) m_{R}-F(R) m_{S}\right\} \\
& \quad+\sum_{R} \sum_{S \neq R} \sum_{T \neq R, S} \frac{J_{R S} J_{S T} J_{T R} F(T) m_{R} m_{S}}{2\left(\omega_{S}-\omega_{T}\right)\left(\omega_{T}-\omega_{R}\right)} \\
& \text { with } \quad F(R)=\left(I_{R}-m_{R}\right)\left(I_{R}+m_{R}+1\right) .
\end{aligned}
$$

When these third-order corrections are included, a much better agreement was found between the experimental and the calculated spectrum of ethyl alcohol. ${ }^{1}$

The first-order perturbed wave functions $v(i)$ are given by

$$
v(i)=u(i)+\sum_{j \neq i} \frac{u(j)\left(j\left|\mathcal{F}^{(1)}\right| i\right)}{E^{(0)}(i)-E^{(0)}(J)},
$$


where $u(i)$ represents the spin functions of the unperturbed system. Upon substituting the values of the matrix elements one finds

$$
\begin{aligned}
& v\left(m_{A}, m_{B}, \cdots\right)=u\left(m_{A}, m_{B}, \cdots\right)-\frac{1}{4} \sum_{R} \sum_{S \neq R} \frac{J_{R S}}{\left(\omega_{R}-\omega_{S}\right)} \\
& \times\left\{G\left(m_{R}\right) G\left(m_{S}+1\right) u\left(\cdots, m_{R}-1, m_{S}+1, \cdots\right)\right. \\
& \text { with } \left.\quad-G\left(m_{R}+1\right) G\left(m_{S}\right) u\left(\cdots, m_{R}+1, m_{S}-1, \cdots\right)\right\}, \\
& G\left(m_{R}\right)=\left\{\left(I_{R}-m_{R}+1\right)\left(I_{R}+m_{R}\right)\right\}^{\frac{1}{2}} .
\end{aligned}
$$

Following the same reasoning outlined in Sec. II we assume that the line intensity is proportional to the square of the matrix elements of

$$
M^{ \pm}=\left(\sum_{R}\right) M_{R^{ \pm}}
$$

Using the first-order wave functions above and keeping only terms to first order when expanding the square, one finds that the line intensity for a transition between a state with energy $E\left(m_{A}, m_{B}, \cdots\right)$ and one with energy $E\left(m_{A}-1, m_{B}, \cdots\right)$ is proportional to

$$
\left(I_{A}-m_{A}+1\right)\left(I_{A}+m_{A}\right)\left\{1-\sum_{R \neq A} \frac{2 J_{A R} m_{R}}{\omega_{A}-\omega_{R}}\right\} .
$$

For only two groups this reduces to Eq. (16) of Sec. II.

\section{APPENDIX III. EFFECT OF APPLYING A SINUSOIDAL VARIATION TO THE MAGNETIC FIELD FOR SMALL RADIO-FREQUENCY FIELDS}

If the applied radio-frequency field is sufficiently small so as not to approach saturation one may replace $M_{z}$ by $M_{0}$ in the first equation of Appendix I. With $m=m_{1} \exp (-i \omega t)$ and $h=h_{1} \exp (-i \omega t)$, this equation becomes

$$
\dot{m}_{1}+i\left(\omega_{A}-\omega\right) m_{1}+m_{1} / T_{2}=i h_{1} M_{0} .
$$

Let the field modulation take the form

$$
\omega_{A}=\omega_{0}+\omega_{m} \cos \left(\omega^{\prime} t\right)
$$

and define $\Delta \omega=\omega_{0}-\omega$. The differential equation has then the solution

$$
m_{1}=i h_{1} M_{0} \int_{-\infty}^{t} \exp \int_{t}^{t^{\prime}}\left\{1 / T_{2}+i \Delta \omega+i \omega_{m} \cos \left(\omega^{\prime} t^{\prime \prime}\right)\right\} d t^{\prime \prime} d t^{\prime} .
$$

If the variation of $\Delta \omega$ is sufficiently slow, one obtains upon integrating within the exponential:

$$
\begin{aligned}
m_{1}=i h_{1} M_{0} \int_{-\infty}^{t}\left[\operatorname { e x p } \left\{\left(1 / T_{2}-i \Delta \omega\right)\left(t^{\prime}-t\right)\right.\right. \\
\left.\left.\quad+i \beta \sin \left(\omega^{\prime} t^{\prime}\right)-i \beta \sin \left(\omega^{\prime} t\right)\right\}\right] d t^{\prime},
\end{aligned}
$$

with $\beta=\omega_{m} / \omega^{\prime}$. This equation may be integrated with the help of the identity

$$
\exp \left\{i \beta \sin \left(\omega^{\prime} t\right)\right\}=\sum_{n=-\infty}^{\infty} J_{n}(\beta) \exp \left(i n \omega^{\prime} t\right)
$$

where $J_{n}(\beta)$ are Bessel functions of the first kind. Upon integrating, one obtains

$$
m_{1}=i h_{1} T_{2} M_{0} \sum_{k=-\infty}^{\infty} \sum_{n=-\infty}^{\infty} \frac{J_{k}(\beta) J_{n}(\beta) \exp \left\{-i(k-n) \omega^{\prime} t\right\}}{1+i\left(\Delta \omega+n \omega^{\prime}\right) T_{2}},
$$

with one of the terms in the summation over $n$ being dominant when $\omega=-n \omega^{\prime}$, where $n$ is any positive or negative integer.

Experimentally we have selected only the component with the frequency of the radio-frequency field $\omega$. Thus the signal output of the $n$th sideband is proportional to $J_{n}^{2}(\beta)$ as is stated in Sec. IV. 


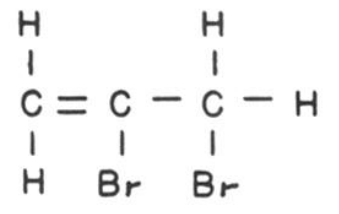

\section{$2,3-D I B R O M O P R O P E N E$}
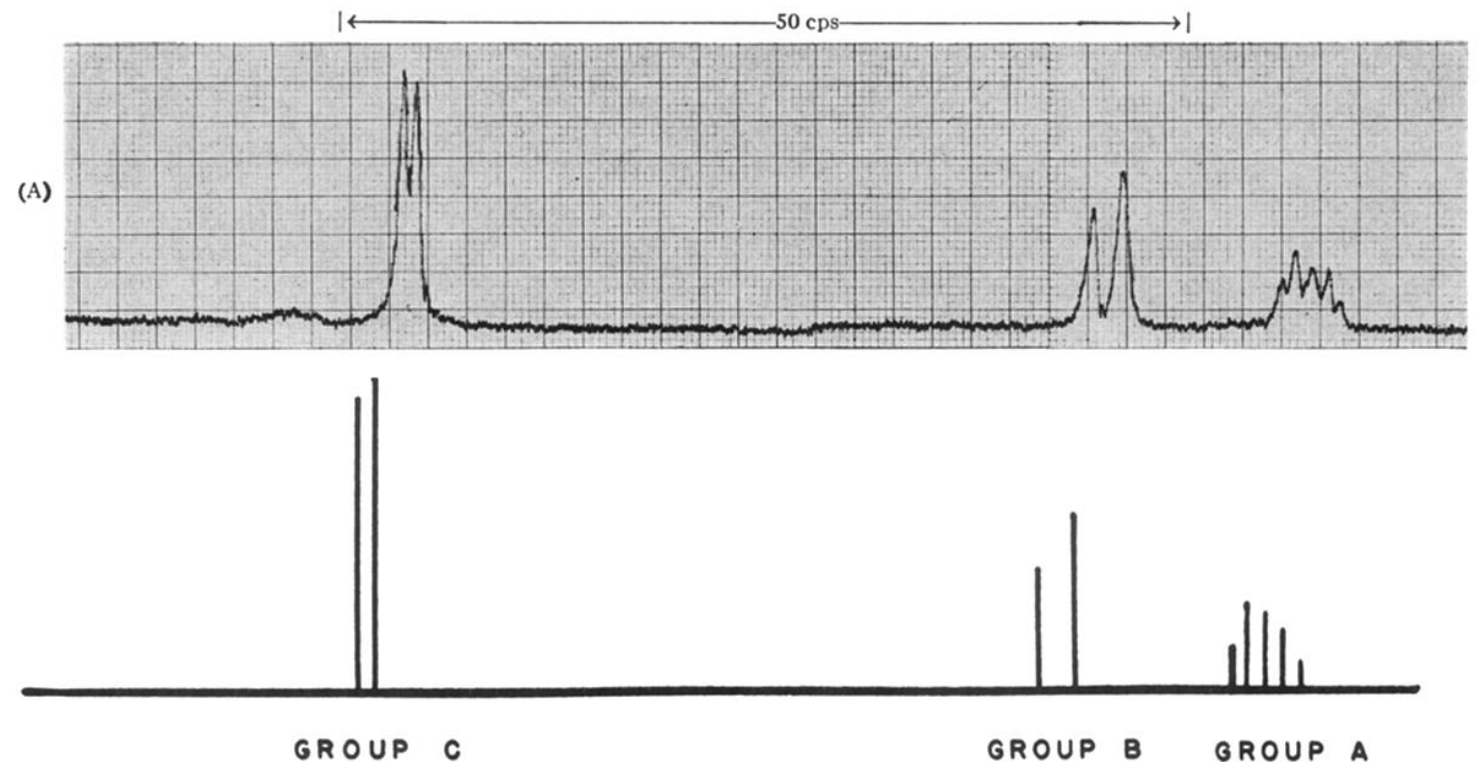

(B)

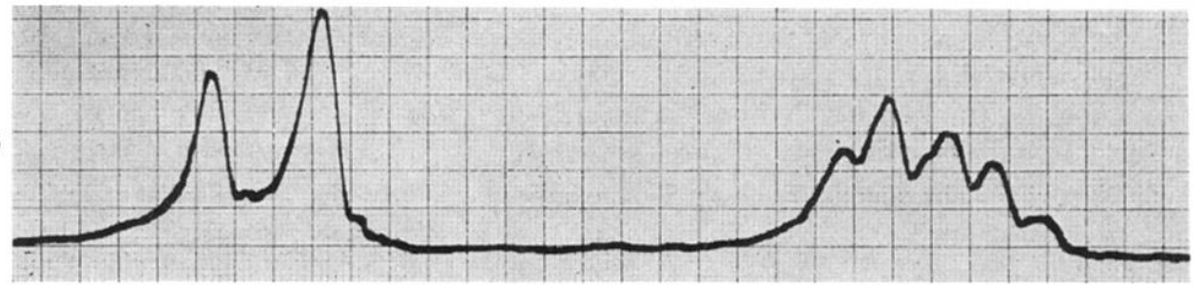

(C)

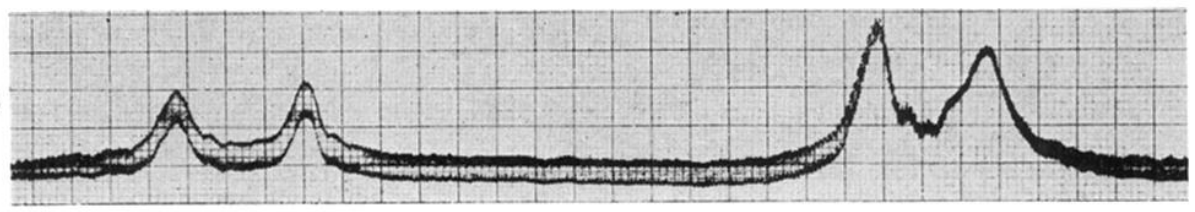

FIg. 10. Spectrum of 2,3-dibromopropene. (A) Total spectrum with the theoretical line spectrum below. The theoretical spectrum was derived using the first-order perturbation theory developed in Appendix II with the results listed in

Table IV. The transitions which occur at the higher frequency appear at the right-hand side of the trace. (B) Spectrum of groups $A$ and $B$ made just before group $C$ was irradiated with a large radio-frequency field. (C) Spectrum of groups $A$ and $B$ investigated with a small radio-frequency field while a large radio-frequency field was applied at a frequency which was lower by 56 cycles per second. Thus while group $A$ was in resonance, group $C$ was being strongly affected by radio-frequency power. 

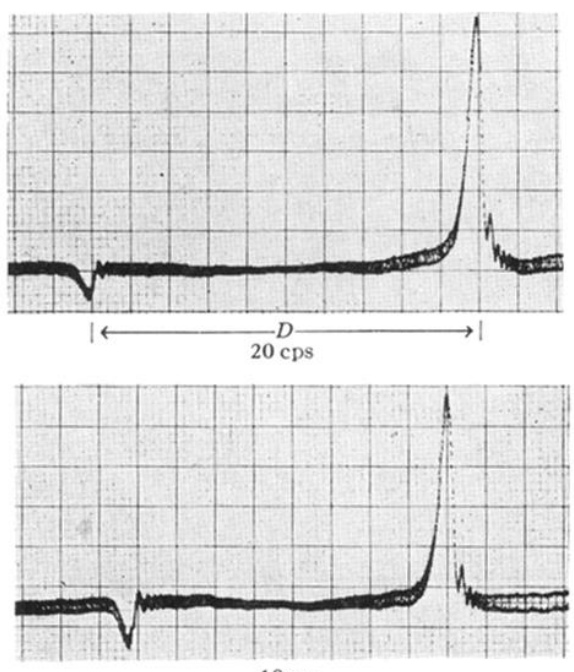

$18 \mathrm{cps}$

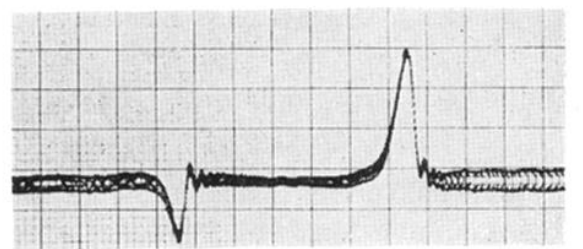

$16 \mathrm{cps}$

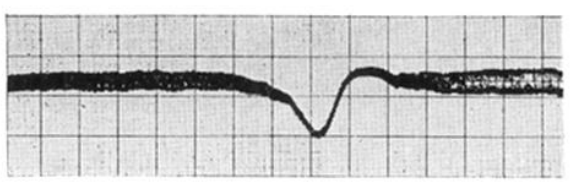

$14 \mathrm{cps}$

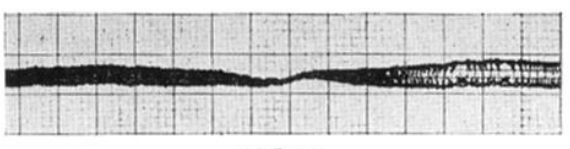

$13.5 \mathrm{cps}$

FIG. 3. Transitions produced by a small radio-frequency field of angular frequency $\omega_{2}$ when water was simultaneously irradiated by a large radio-frequency field of angular frequency $\omega_{1}$. The number below each trace is the frequency difference of the two fields $\left(\omega_{2}-\omega_{1}\right) /(2 \pi)$. The relative magnitude of the two signals is here immaterial and distorted since the receiver was overoaded by the large radio-frequency field. 

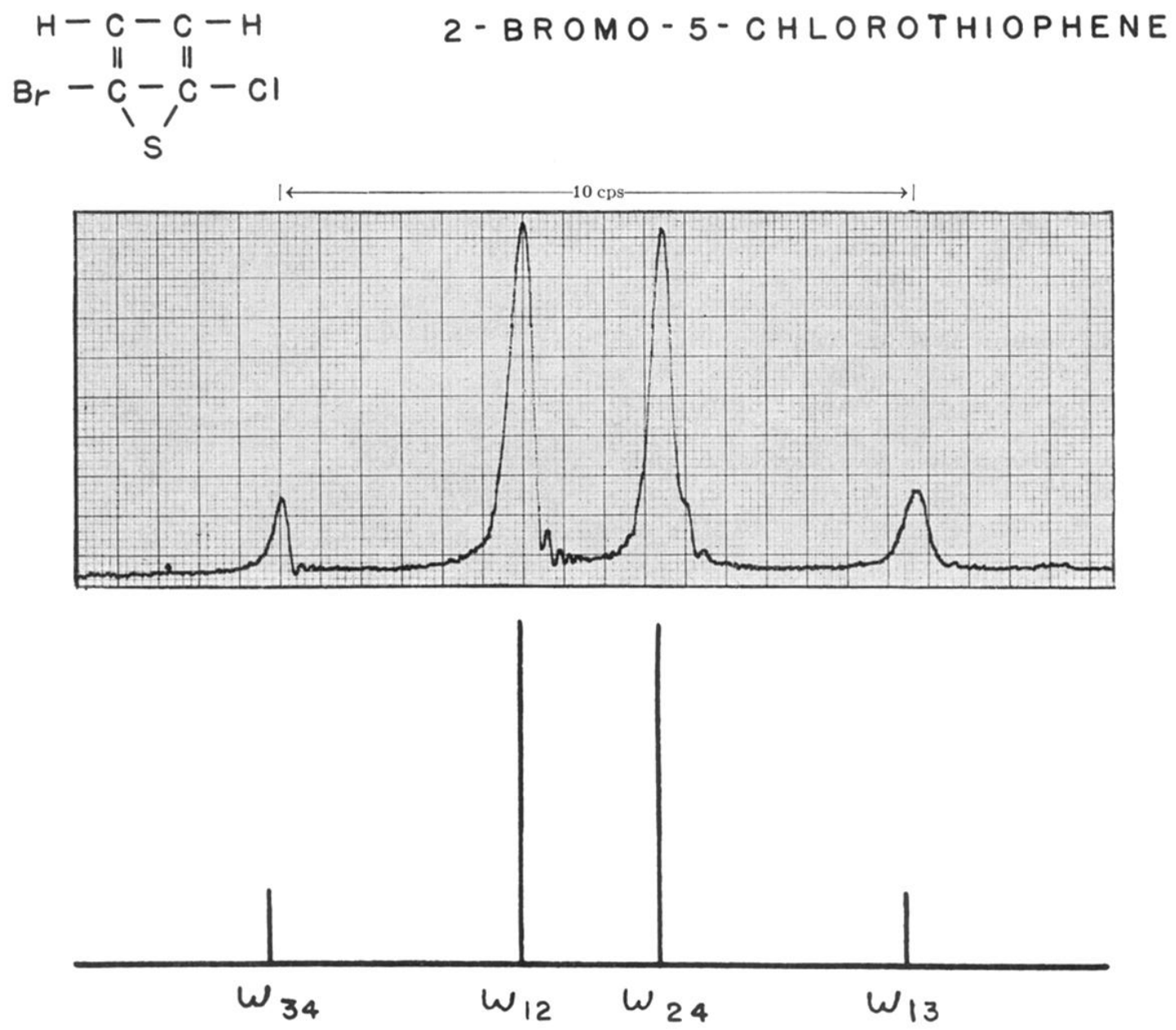

Fig. 5. Spectrum of 2-bromo-5-chlorothiophene. The theoretical spectrum below was calculated from the expressions given in Table I with the ratio $J / \delta=0.835$. The labeling of the lines is identical to that given in Table I. 


$$
\begin{array}{cl}
C I \\
C \\
I \\
C
\end{array}-\underset{H}{C}=0
$$

\section{DICHLOROACETALDEHYDE}
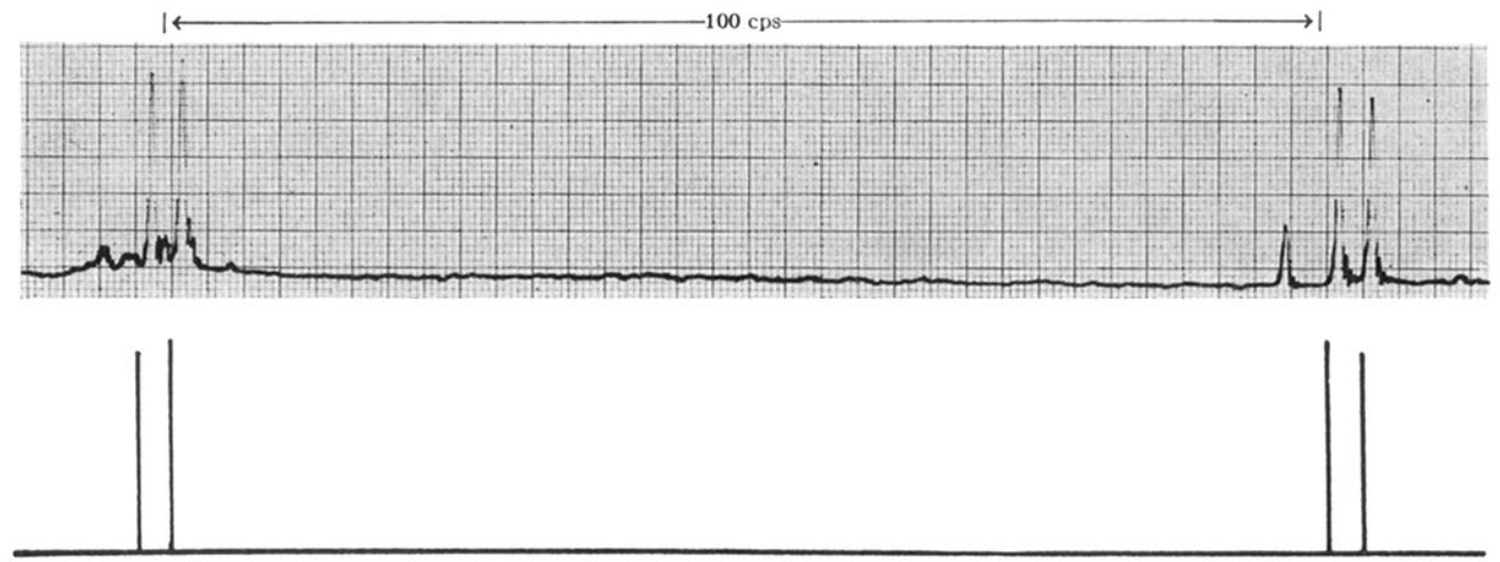

Fig. 6. Spectrum of dichloroacetaldehyde. The small peaks are due to impurities. The theoretical spectrum was calculated assuming that $J / \delta=0.029$. 


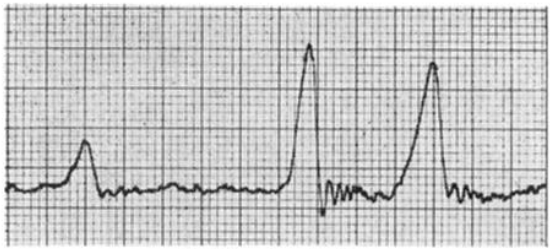

Without radiation

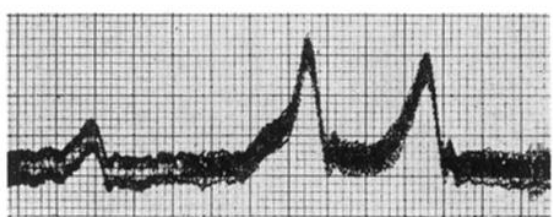

$110 \mathrm{cps}$

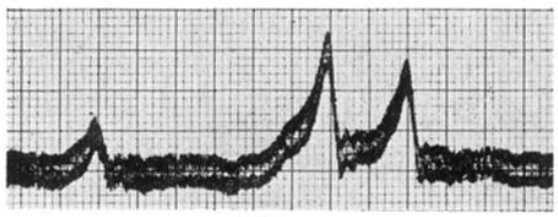

$105 \mathrm{cps}$

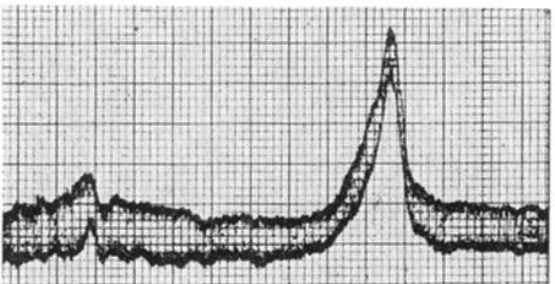

$100 \mathrm{cps}$

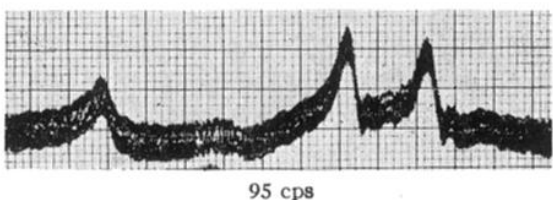

FIG. 7. Resonance in the group appearing at the lower field in dichloroacetaldehyde produced by a small radio-frequency field of angular frequency $\omega_{2}$, while a strong radio-frequency field of angular frequency $\omega_{1}$ is applied near the resonance frequency of the other group. The amplitude of the strong field is about $1 \frac{1}{2}$ milligauss. The frequency difference of the two fields $\left(\omega_{2}-\omega_{1}\right) /(2 \pi)$ is given below each trace. The small peak on the left of each trace is the result of an impurity, and it may be used as a reference mark. 
<smiles>ClCC(Cl)Cl</smiles>

\section{I,1,2 - TRICHLOROETHANE}

(A)
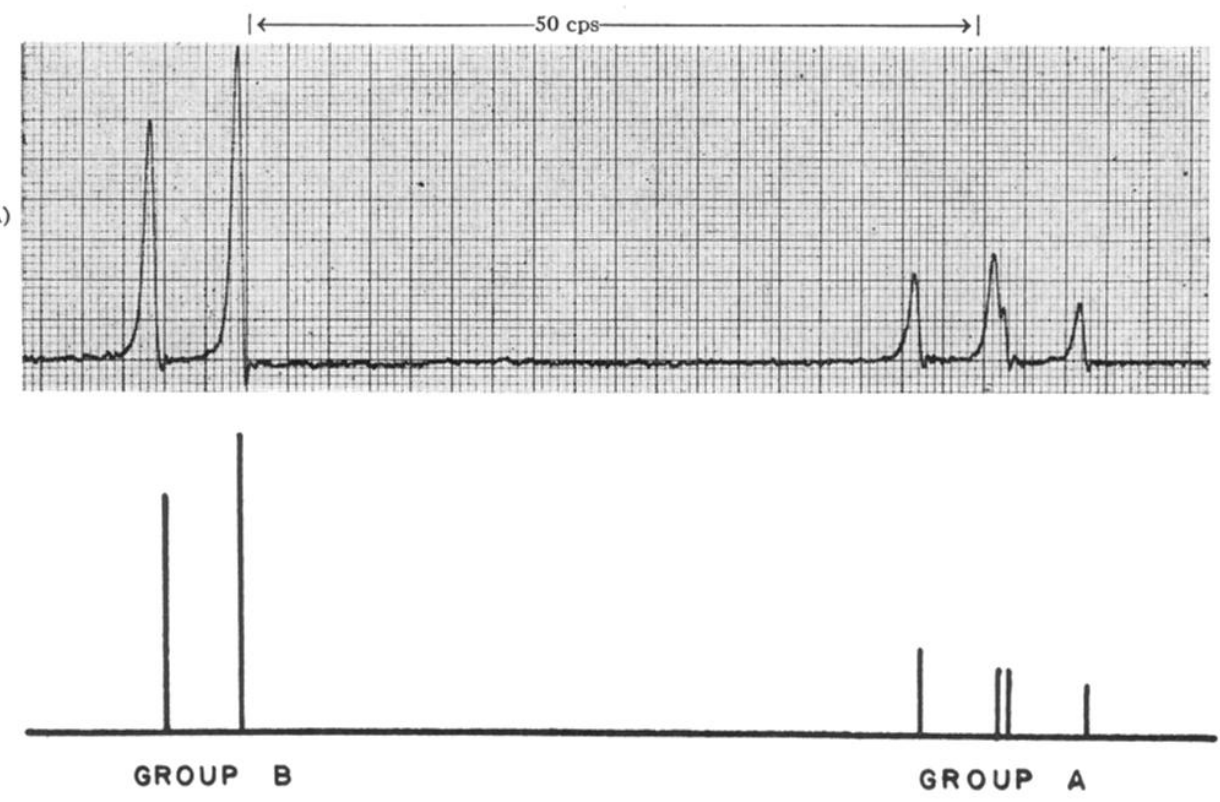

(B)
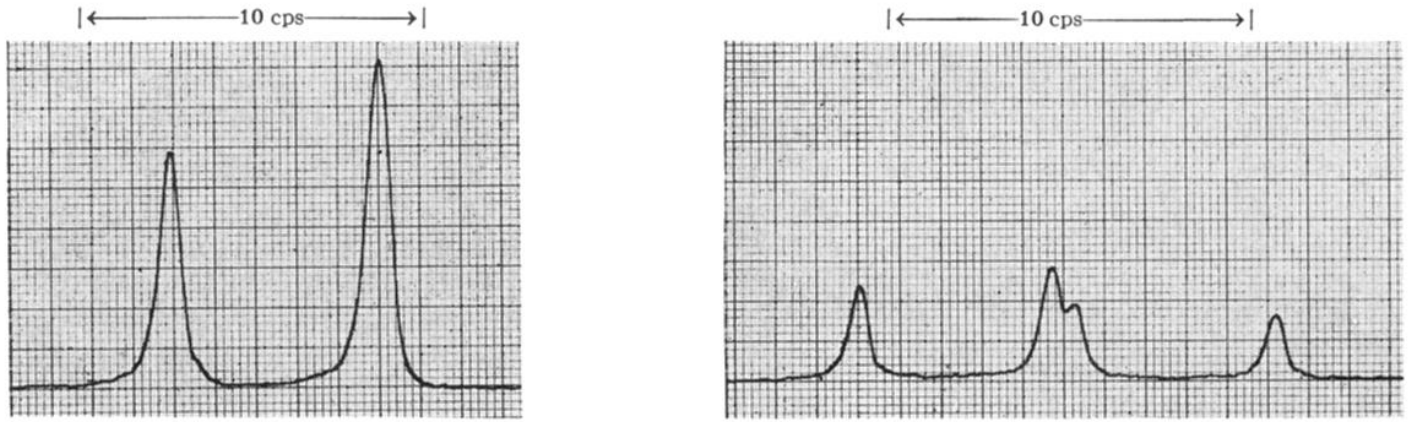

(C)

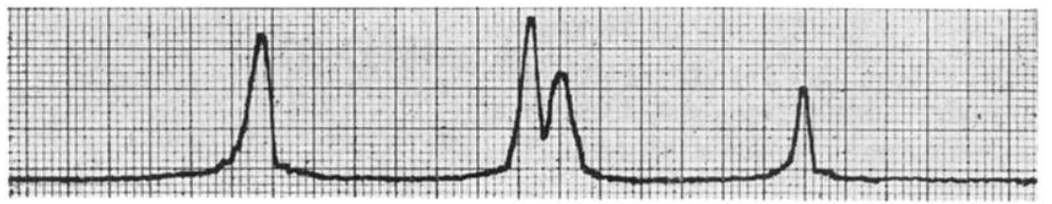

Fig. 8. Spectrum of 1,1,2-trichloroethane. (A) Total spectrum with theoretical line spectrum below. The theoretical spectrum was derived from the expression given in Table II assuming $J / \delta=0.105$. The lines occuring at the higher frequency appear at the right-hand side of the trace. Although each of the two lines in group $B$ should be doubled, the splitting is too small to be resolved. Single lines are therefore drawn in the theoretical intensity pattern each with a length equal to the sum of the lengths of the two lines which they represent. (B) The spectrum of each group obtained by sweeping the magnetic field more slowly. (C) The spectrum of group $A$ under better resolution; however, the right-hand peak appears somewhat distorted, probably because of nonlinearities in the rate of sweep of the magnetic field. In this trace one of the two central lines is associated with the state $I_{B}=0, m_{B}=0$ of group $B$ and it definitely appears narrower as well as higher than the adjacent other line, associated with the state $I_{B}=1$, $m_{B}=0$. 


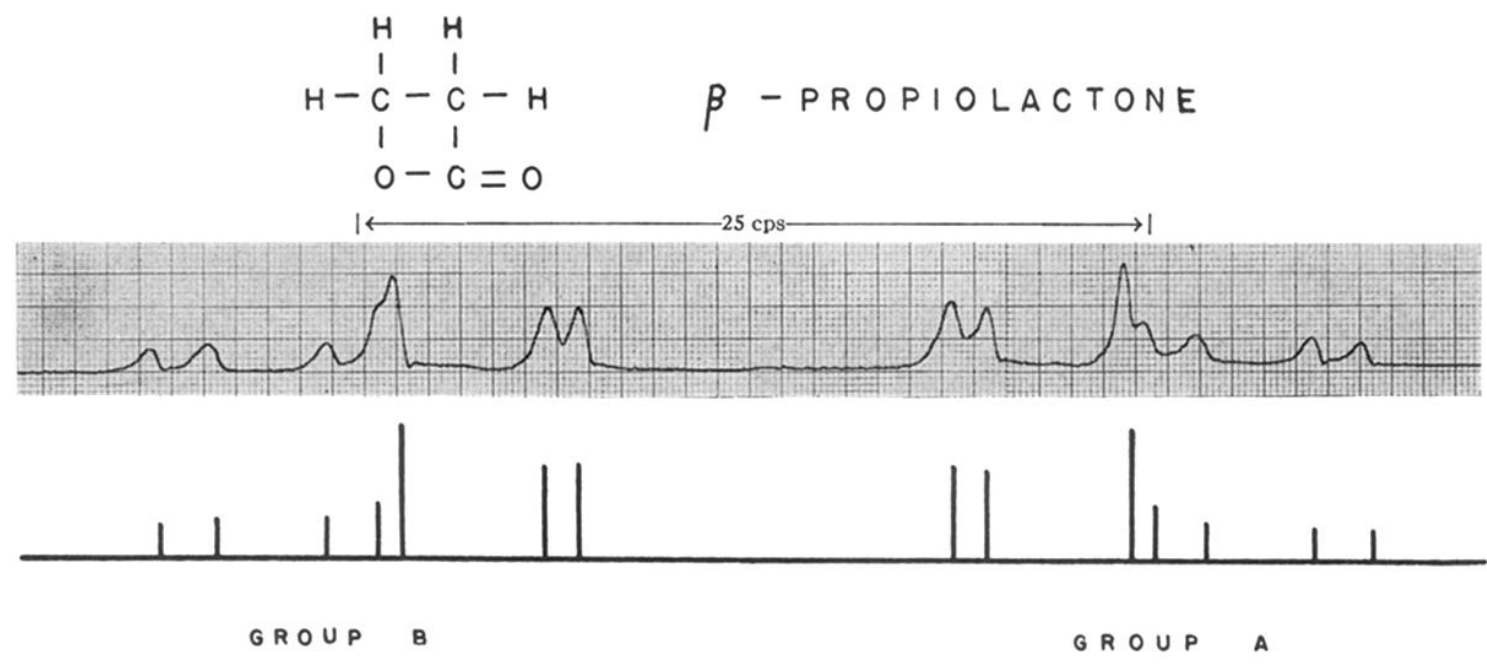

FIG. 9. Spectrum of $\beta$-propiolactone. The theoretical line spectrum below was calculated in the manner outlined in Sec. II for the case of $J / \delta=0.265$ with the results given in Table III. 